\title{
Mixed structures on fundamental groups
}

\author{
Jörg Wildeshaus \\ Mathematisches Institut \\ der Universität Münster \\ Einsteinstr. 62 \\ D-48149 Münster*
}

*current address: Dept. of Math. Sciences, South Road, GB-Durham DH1 3LE 


\section{Introduction}

Let $X$ be a smooth, separated, geometrically connected scheme over a number field $k$. In [D4], Deligne constructed a mixed system of realizations on the completed groups rings of the topological fundamental groups of $X$ in the various (Betti, de Rham, ...) contexts. He did so under the very restrictive hypothesis that the $H^{1}\left(X_{\sigma}, \mathbb{Q}\right)$ be of Hodge type $(1,1)$ for any embedding $\sigma: k \hookrightarrow \mathbb{C}$.

In his attempt to understand and generalize Beilinson's work on polylogarithms $([\mathrm{B}])$, the present author soon realized that it would be of vital importance to further develop Deligne's program - excluding the crystalline version - by not only removing the above hypothesis but also by considering the relative situation, i.e., the case of a suitably regular morphism $X \rightarrow Y$. Indeed, the central, if elementary insight is already contained in Proposition 1.4. In the applications we have in mind it states that the injectivity of the natural morphism of the pro-unipotent envelope of the fundamental group to the Tannaka dual of some category of mixed sheaves on $X$ is equivalent to the existence of a mixed sheaf structure on the completed group ring itself. So the desire to describe a part of this Tannaka dual necessitates the study of mixed structures on fundamental groups.

The main results of this work are those of $\S 3$, and Theorem 4.3. We chose to discuss the absolute situation separately $(\S 2)$, hoping that this would clarify the exposition by focusing on the actual construction of the mixed structures, which in the relative case are then defined fibrewise. Also, this reflects the development of ideas: the rather general results 1.4 and 1.5 were modelled after the classification theorem for admissible unipotent variations of Hodge structure ([HZ1], Theorem 1.6), which we recall in 2.6. So the main results of $\S 2(2.9$, 2.12 ) should be seen as analogues of this theorem in the $\lambda$-adic and "mixed realizations" settings, while the results of $\S 3$ are the respective relative versions. Likewise, Theorem 4.3 should be regarded as a generalization of [CH], Theorem 12.1 .

As an immediate consequence of the classification theorems for relatively unipotent sheaves (Corollaries 3.2.ii) and 3.4.ii), Theorem 3.6.c)), we establish a certain universal property of our "generic relatively unipotent sheaf" (Theorems 3.5 and 3.6.d)). It is this universal property, which will turn out to be a most use- 
ful device both in the study of the functor "canonical construction" of sheaves on Shimura varieties from representations of the group underlying the Shimura data, and the development of the general theory of polylogarithmic extensions. In the case of a family of elliptic curves, the result appears already in [BL], 1.2.8.b).

We conclude by an application of 4.3 in a rather special case (Corollary 4.4). While this may appear as an anticlimax at first sight, this computation is again motivated directly by the author's interest in polylogarithms. In his attempt to generalize the work of Beilinson, Deligne and Levin ([BD], [BL]) to the context of mixed Shimura varieties ([W]), the computation in 4.4 constitutes the first step toward the definition of the polylogarithmic extension. Details will be published separately.

In our work, motivic sheaves are hardly ever mentioned although they certainly motivate our study to a large extent. In fact, as a very first step in the direction of a K-theoretical construction of polylogarithms, one should convince oneself that the "generic relatively unipotent sheaves" of $\S 3$ (renamed "logarithmic sheaves" in the context of Shimura varieties) are actually of motivic, or geometric origin. In the absolute case $(\S 2)$, this follows (with a suitable definition of the term "of geometric origin") from work of Wojtkowiak ([Wo1], [Wo2]).

This article is a revised version of the first chapter of my doctoral thesis ([W]). It is a pleasure to thank C. Deninger for introducing me to Beilinson's ideas and for his generosity and constant support. I am obliged to A. Beilinson for kindly supplying me with copies of [BLpp] and [BLp], thus enabling me to work with the material long before is was published. I tried occasionally to indicate the impact that the concepts developed in [B], [BLpp] and [BLp] had on this work.

I am grateful to F. Oort and J. Stienstra for the invitation to the "intercity seminarium algebra en meetkunde" in March 1992, and to J. Coates for the invitation to Cambridge in Spring 1993. Both occasions proved to be very stimulating. Also, I would like to thank T. Scholl for the invitation to Durham in 1994, where all results concerning the de Rham version of the generic sheaf were found. 
Last, but not least, I am most deeply indebted to Mrs. G. Weckermann for transforming my manuscript into an excellent $\mathrm{T}_{\mathrm{E}} \mathrm{X}$-file. 


\section{$\S 1$ Review of neutral Tannakian categories and pro-unipotent groups}

We follow the treatment of $[\mathrm{DM}], \S \S 1-2$.

Let $F$ be a field, $P / F$ an affine group scheme. Then the category $\mathcal{C}:=\operatorname{Rep}_{F}(P)$ of finite-dimensional representations of $P$ over $F$ is a neutral Tannakian category over $F$, i.e.:

i) it is abelian.

ii) it is a tensor category, i.e., there exists a functor

$$
\otimes: \mathcal{C} \times \mathcal{C} \longrightarrow \mathcal{C}
$$

together with associativity and commutativity constraints subject to the following condition:

for $X, Y, Z \in \mathcal{C}$

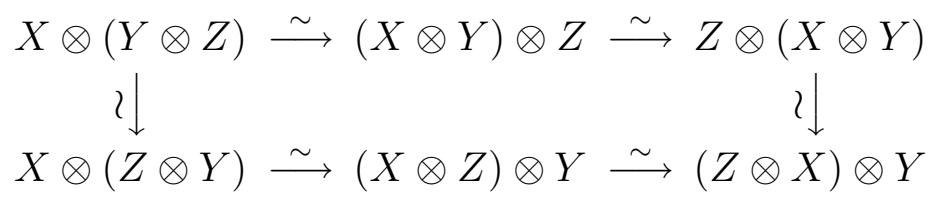

is commutative.

Furthermore, there is a left neutral element $U$ for $\otimes$.

iii) it is rigid, i.e., there is a functor

$$
\underline{\text { Hom }}: \mathcal{C} \times \mathcal{C} \longrightarrow \mathcal{C}
$$

representing

$$
T \mapsto \operatorname{Hom}_{\mathcal{C}}\left(T \otimes{ }_{-},{ }_{-}\right),
$$

such that every object of $\mathcal{C}$ is reflexive, i.e., canonically isomorphic to its bidual and such that

$$
\underline{\operatorname{Hom}}\left(X_{1}, Y_{1}\right) \otimes \underline{\operatorname{Hom}}\left(X_{2}, Y_{2}\right)=\underline{\operatorname{Hom}}\left(X_{1} \otimes X_{2}, Y_{1} \otimes Y_{2}\right), X_{i}, Y_{i} \in \mathcal{C} .
$$

iv) $F=\operatorname{End}_{\mathcal{C}}(U)$, and there is a fibre functor, i.e., an exact faithful $F$-linear tensor functor

$$
\omega: \mathcal{C} \longrightarrow \operatorname{Vec}_{F} .^{\dagger}
$$

Here, $\operatorname{Vec}_{F}$ is the category of finite-dimensional vector spaces over $F$.

\footnotetext{
${ }^{\dagger} \omega$ is not part of the data!
} 
The Main Theorem of the Tannakian formalism ([DM], Theorem 2.11) gives a converse of the above construction:

Theorem 1.1: Let $\mathcal{C}$ be a neutral Tannakian category over $F$. Then every fibre functor $\omega$ defines an equivalence of tensor categories

$$
\mathcal{C} \stackrel{\sim}{\longrightarrow} \operatorname{Rep}_{F}(P)
$$

for some affine group scheme $P$ over $F$.

We refer to $P$ as the Tannaka dual of $\mathcal{C}$ with respect to $\omega$.

$P$ represents a certain functor $\underline{\mathrm{Aut}}^{\otimes}(\omega)$ of affine $F$-schemes. This shows that any $F$-linear tensor functor $\mathcal{C} \rightarrow \mathcal{C}^{\prime}$ of neutral Tannakian categories compatible with fixed choices of fibre functors $\omega$ and $\omega^{\prime}$ comes from a morphism $P^{\prime} \rightarrow P$ of the associated Tannaka duals.

It is possible to recover properties of $P$ and of morphisms $P^{\prime} \stackrel{\pi}{\longrightarrow} P$ from those of $\operatorname{Rep}_{F}(P)$ and of $\pi^{*}: \operatorname{Rep}_{F}(P) \rightarrow \operatorname{Rep}_{F}\left(P^{\prime}\right)$ :

Proposition 1.2: ([DM], Proposition 2.21.)

a) $\pi$ is faithfully flat if and only if $\pi^{*}$ is fully faithful and every subobject of $\pi^{*}(X), X \in \operatorname{Rep}_{F}(P)$, is isomorphic to the image of a subobject of $X$.

b) $\pi$ is a closed immersion if and only if every object of $\operatorname{Rep}_{F}\left(P^{\prime}\right)$ is isomorphic to a subquotient of an object of the form $\pi^{*}(X), X \in \operatorname{Rep}_{F}(P)$.

As any morphism of $F$-Hopf algebras factors uniquely into a surjection followed by an injection, any morphism of affine group schemes over $F$ factors uniquely into a faithfully flat morphism followed by a closed immersion.

In particular, a morphism of affine group schemes over $F$ is a closed immersion if and only if it is injective on points.

Quite often, we shall have to study morphisms of groups with pro-unipotent kernel:

Lemma 1.3: Let $P \stackrel{\pi}{\longrightarrow} G$ be a faithfully flat morphism, $W:=\operatorname{ker}(\pi)$.

Then $W$ is pro-unipotent if and only if every nonzero object $X \in \operatorname{Rep}_{F}(P)$ has a nonzero subobject of the form $\pi^{*}(Y)$, where $Y \in \operatorname{Rep}_{F}(G)$.

Proof: If $W$ is pro-unipotent then $0 \neq X^{W}$ for $0 \neq X \in \operatorname{Rep}_{F}(P)$. 
Conversely, assume every $X \in \operatorname{Rep}_{F}(P)$ has a finite filtration

$$
0=X_{0} \subset X_{1} \subset \ldots \subset X_{n}=X
$$

whose graded objects are trivial as $W$-modules. Then the same is true for any $W$-subquotient of $X$.

From 1.2.b) we conclude that every nonzero $X \in \operatorname{Rep}_{F}(W)$ has non-trivial invariants.

q.e.d.

Proposition 1.4: In the situation of 1.3, assume $W=\operatorname{ker}(\pi)$ is pro-unipotent. Let $\bar{W} \stackrel{\eta}{\longrightarrow} W$ be a morphism of pro-unipotent groups.

a) $\eta$ is faithfully flat if and only if the following holds: for any $X \in \operatorname{Rep}_{F}(P), X^{\bar{W}}$ is the largest subobject of $X$ lying in $\pi^{*}\left(\operatorname{Rep}_{F}(G)\right)$.

b) Assume $\pi$ admits a right inverse, $\eta$ is faithfully flat and $\operatorname{char}(F)=0$. Then $\eta$ is an isomorphism if and only if there is an action of $P$ on $\hat{\mathfrak{U}}(\mathrm{Lie} \bar{W})$ such that the associated action of Lie $P$ extends the multiplication by $\operatorname{Lie} \bar{W} \subset \hat{\mathfrak{U}}(\operatorname{Lie} \bar{W})$. Here, $\hat{\mathfrak{U}}(\mathrm{Lie} \bar{W})$ is defined as follows:

write $\operatorname{Lie} \bar{W}=\underset{j}{\lim } \mathfrak{w}_{j}$ as the projective limit of its finite-dimensional quotient Lie algebras.

Then $\hat{\mathfrak{U}}(\operatorname{Lie} \bar{W}):={\underset{j}{j}}_{\lim } \hat{\mathfrak{U}}\left(\mathfrak{w}_{j}\right)$, where $\hat{\mathfrak{U}}\left(\mathfrak{w}_{j}\right)$ is the completion of the universal enveloping algebra of $\mathfrak{w}_{j}$ with respect to the augmentation ideal $\mathfrak{a}_{j}$.

The action of $P$ is supposed to be such that the algebraic $P$-representation spaces are cofinal in the projective system $\left(\hat{\mathfrak{U}}\left(\mathfrak{w}_{j}\right) / \mathfrak{a}_{j}^{n} \mid j \in J, n \in \mathbb{N}\right) .^{\dagger}$

Proof: a) Nothing changes if we replace $\bar{W}$ by $\eta(\bar{W})$, which is a closed subgroup of $W$. Also, we may suppose that everything is algebraic.

By one of Chevalley's Theorems ([Hum1],

Theorem 11.2) there is a representation $X$ of $P$ and a one-dimensional subspace $L \subset X$ such that

$$
\bar{W}=\operatorname{Stab}_{P}(L) .
$$

Since $\bar{W}$ is unipotent, $\bar{W}$ acts trivially on $L$, i.e., $L \subset X^{\bar{W}}$. By hypothesis, $W$ also acts trivially on $L$. So $\bar{W}$ and $W$ must be equal.

b) Since $\operatorname{char}(F)=0$, there is an equivalence of categories ([DG], IV, $\S 2$, Corollaire 4.5.b))

$$
\operatorname{Rep}_{F}(\bar{W}) \stackrel{\sim}{\longrightarrow} \operatorname{Mod}_{\mathfrak{\mathcal { U }}(\operatorname{Lie} \bar{W})}^{\mathrm{fin}},
$$

\footnotetext{
${ }^{\dagger}$ By definition, $\mathbb{N}$ is the set of positive integers, and $\mathbb{N}_{0}$ is the set of non-negative integers.
} 
the latter denoting the category of finite-dimensional $\hat{\mathfrak{U}}(\mathrm{Lie} \bar{W})$-modules such that the action is continuous with respect to the discrete topology on the module and the inverse limit topology on $\hat{\mathfrak{U}}(\operatorname{Lie} \bar{W})$. The algebra $\hat{\mathfrak{U}}(\mathrm{Lie} \bar{W})$ acting on itself by multiplication is a pro-object of $\operatorname{Rep}_{F}(\bar{W})$, and every $X \in \operatorname{Rep}_{F}(\bar{W})$ is a subquotient of a finite number of copies of $\hat{\mathfrak{U}}(\operatorname{Lie} \bar{W})$. So if this representation of $\bar{W}$ can be extended to $P$, we may apply 1.2 .b) to conclude that $\bar{W} \rightarrow P$ is a closed immersion.

Conversely, if $\bar{W}=W$, we let $G$ act on $\hat{\mathfrak{U}}(\mathrm{Lie} W)$ by conjugation, using the section of $\pi$. This, together with the above action of $W$, defines the desired action of $P=W \times G$.

q.e.d.

Remark: There is always the canonical action "Ad" of $P$ on $\hat{\mathfrak{U}}(\operatorname{Lie} W)$, but the associated action of Lie $P$ extends the action "ad" of Lie $W$, which we shall have to distinguish carefully from the multiplication by LieW. "ad" is a representation of $\operatorname{Lie}(W / Z(W))$, which in general won’t generate $\operatorname{Mod}_{\hat{\mathfrak{U}}(\operatorname{Lie} W)}^{\mathrm{fin}}$.

Lemma 1.5: Assume $\operatorname{char}(F)=0$ and $P=W \times G$ with a pro-unipotent group $W$. Then $\operatorname{Rep}_{F}(P)$ is equivalent to the category of finite-dimensional discrete $\hat{\mathfrak{U}}($ Lie $W)$-modules $X$ equipped with a representation of $G$ such that the morphism

$$
\hat{\mathfrak{U}}(\operatorname{Lie} W) \longrightarrow \operatorname{End}_{F}(X)
$$

is $G$-equivariant, $G$ acting on $\hat{\mathfrak{U}}(\mathrm{Lie} W)$ by conjugation.

Proof: straightforward.

q.e.d.

In the rest of this paragraph, we shall discuss several methods of calculating Yoneda-Ext groups of representations of a pro-unipotent group scheme over a field of characteristic 0 . The results won't be needed until $\S 4$, and the reader is invited to ignore them until then.

So let $\operatorname{char}(F)=0$,

$$
\begin{aligned}
& W=\lim _{j \in \mathbb{N}} W_{j} \quad \text { a pro-unipotent group scheme over } F \text {, which is a countable } \\
& \text { projective limit of unipotent group schemes } W_{j} \text {, } \\
& \mathfrak{w}=\lim _{j} \mathfrak{w}_{j} \quad \text { its Lie-algebra, } \\
& \mathfrak{U}\left(\mathfrak{w}_{j}\right) \text { the universal enveloping algebra, } \\
& \hat{\mathfrak{U}}\left(\mathfrak{w}_{j}\right) \text { the completion with respect to the augmentation ideal } \mathfrak{a}_{j},
\end{aligned}
$$




$$
\begin{aligned}
\hat{\mathfrak{U}}(\mathfrak{w}) & :=\underbrace{\lim }_{j} \hat{\mathfrak{U}}\left(\mathfrak{w}_{j}\right)=\underbrace{\lim }_{j, n} \mathfrak{U}\left(\mathfrak{w}_{j}\right) / \mathfrak{a}_{j}^{n}=\underbrace{\lim }_{n} \mathfrak{U}\left(\mathfrak{w}_{n}\right) / \mathfrak{a}_{n}^{n}, \\
\mathfrak{a} & :=\underbrace{\lim }_{j} \hat{\mathfrak{a}}_{j} \text { the augmentation ideal of } \hat{\mathfrak{U}}(\mathfrak{w}) .
\end{aligned}
$$

Following $[\mathrm{Ho}]$, we define

$$
\begin{aligned}
\operatorname{Rat}(W):= & \operatorname{Ind}-\operatorname{Rep}_{F}(W)= \\
& \{\text { discrete modules under } \hat{\mathfrak{U}}(\mathfrak{w}) \text { with continuous action }\} .
\end{aligned}
$$

Proceeding dually to [H1], $\S 3$, we define $R(W)$ as the full subcategory of $\operatorname{Rat}(W)$ of modules of countable dimension. So

$$
\begin{aligned}
R(W)=\{X \in \operatorname{Rat}(X) \mid & X \text { is the union of countably many } \\
& \text { algebraic representation spaces of } W\} .
\end{aligned}
$$

$R(W)$ and $\operatorname{Rat}(W)$ are closed under $\otimes_{F}$, and the topological dual of $\hat{\mathfrak{U}}(\mathfrak{w})$,

$$
(\hat{\mathfrak{U}}(\mathfrak{w}))^{\stackrel{c}{\vee}}:=\operatorname{Hom}_{F, \text { cont. }}(\hat{\mathfrak{U}}(\mathfrak{w}), F)=\underset{\overrightarrow{j, n}}{\lim }\left(\mathfrak{U}\left(\mathfrak{w}_{j}\right) / \mathfrak{a}_{j}^{n}\right)^{\vee}
$$

is an object of $R(W)$. Here, the action of $\hat{\mathfrak{U}}(\mathfrak{w})$ on itself is given by multiplication.

Consider the functor $*: \operatorname{Mod}_{W(F)} \rightarrow \operatorname{Rat}(W):$

$$
M \mapsto \text { the maximal rational submodule of } M \text {. }
$$

The functor *, being the right adjoint to the forgetful functor, which is exact, maps injectives to injectives.

\section{Proposition 1.6:}

a) If $M \in \operatorname{Mod}_{W(F)}$ is projective, then $M^{\vee}:=\operatorname{Hom}_{F}(M, F) \in \operatorname{Mod}_{W(F)}$ is injective.

b) $R(W)$ and $\operatorname{Rat}(W)$ are abelian subcategories of $\operatorname{Mod}_{W(F)}$ with sufficiently many injectives.

c) The inclusions $\operatorname{Rep}_{F}(W) \hookrightarrow R(W)$ and $R(W) \hookrightarrow \operatorname{Rat}(W)$ respect YonedaExt groups. In particular, for any $X \in \operatorname{Rep}_{F}(W)$, Hochschild cohomology $H^{\cdot}(W, X)=\operatorname{Ext}_{\operatorname{Rat}(W)}^{\prime}(F, X)$ coincides with $\operatorname{Ext}_{\operatorname{Rep}_{F}(W)}(F, X)$.

Proof: a) straightforward.

b) "abelian": easy. 
"enough injectives" for Rat $(W)$ : (compare [Ho], Proposition 2.1.)

$\operatorname{Mod}_{W(F)}$ has enough injectives. Given $X \in \operatorname{Rat}(W)$, choose a monomorphism $X \hookrightarrow I$ into an injective object $I \in \operatorname{Mod}_{W(F)}$. This map must factor through $I^{*}$.

"enough injectives" for $R(W)$ : (compare [H1], Proposition 4.8, Lemma 4.9.)

1) In $R(1)$ every element is injective.

2) The forgetful functor $V: R(W) \rightarrow R(1)$ has the right adjoint

$$
R(1) \rightarrow R(W), Y \mapsto(\hat{\mathfrak{U}}(\mathfrak{w}))^{\vee} \otimes_{F} Y:
$$

Let $X \in R(W), Y \in R(1), X=\bigcup_{i \in \mathbb{N}} X_{i}$ such that $\operatorname{dim}_{F} X_{i}<\infty$. Then

$$
\begin{aligned}
& \operatorname{Hom}_{R(W)}\left(X,(\hat{\mathfrak{U}}(\mathfrak{w}))^{\vee} \vee \otimes_{F} Y\right)=\underbrace{\lim }_{i}\left(\operatorname{Hom}_{R(W)}\left(X_{i},(\hat{\mathfrak{U}}(\mathfrak{w}))^{\vee}\right) \otimes_{F} Y\right) \\
& =\underbrace{}_{i} \lim _{i}\left(\operatorname{Hom}_{\hat{\mathfrak{U}}(\mathfrak{w}), \text { cont. }}\left(\hat{\mathfrak{U}}(\mathfrak{w}), X_{i}^{\vee}\right) \otimes_{F} Y\right) \\
& =\lim _{i} \operatorname{Hom}_{F}\left(X_{i}, Y\right) \\
& =\operatorname{Hom}_{R(1)}(V(X), Y) \text {. }
\end{aligned}
$$

Since $V$ is exact, its adjoint maps injectives to injectives.

3) For all $X \in R(W)$, the adjunction morphism

$$
X \longrightarrow(\hat{\mathfrak{U}}(\mathfrak{w}))^{\vee} \otimes_{F} V(X)
$$

is injective.

c) For a quite general study of the question, "when are we allowed to calculate cohomological derived functors on a category in its Ind-category?", see [Hub], $\S 2$, especially Theorem 2.6.

For the special cohomological derived functor "Yoneda-Ext", we refer to the following lemma.

q.e.d.

Lemma 1.7: Let $\mathcal{A}_{1} \subset \mathcal{A}_{2} \subset \mathcal{A}_{3}$ be fully faithful, exact inclusions of abelian categories such that

i) $\mathcal{A}_{1}$ is the category of noetherian objects of $\mathcal{A}_{3}$.

ii) Every object in $\mathcal{A}_{2}$ is a countable direct limit of objects of $\mathcal{A}_{1}$, and every countable direct system in $\mathcal{A}_{1}$ has a limit in $\mathcal{A}_{2}$. 
iii) Every object in $\mathcal{A}_{3}$ is a direct limit of objects in $\mathcal{A}_{1}$.

Then the inclusions $\mathcal{A}_{1} \hookrightarrow \mathcal{A}_{2}$ and $\mathcal{A}_{2} \hookrightarrow \mathcal{A}_{3}$ respect Yoneda-Ext groups.

Proof: (sketch)

surjectivity: the essential point is that if $A$ and $B$ are objects of $\mathcal{A}_{1}$ and $\mathcal{A}_{3}$ respectively, and if

$$
0 \rightarrow B \rightarrow E \rightarrow A \rightarrow 0
$$

is exact in $\mathcal{A}_{3}$, then this sequence is the push-out of a sequence in $\mathcal{A}_{1}$.

Using this, for any exact sequence

$$
0 \rightarrow B \rightarrow E_{1} \rightarrow \ldots \rightarrow E_{n} \rightarrow A \rightarrow 0
$$

in $\mathcal{A}_{3}$ with $A, B$ in $\mathcal{A}_{1}$, one constructs a sequence

$$
0 \rightarrow B \rightarrow E_{1}^{\prime} \rightarrow \ldots \rightarrow E_{n}^{\prime} \rightarrow A \rightarrow 0
$$

in $\mathcal{A}_{1}$ and a morphism of this $n$-extension into the above, i.e., an elementary equivalence. $(*)$

This proves the surjectivity for the relative situation $\mathcal{A}_{1} \hookrightarrow \mathcal{A}_{3}$. Using this, the same procedure shows the analogous statement for $\mathcal{A}_{2} \hookrightarrow \mathcal{A}_{3}$.

injectivity: the main step in the proof is the following observation: if $\mathcal{E} \rightarrow \mathcal{E}_{1} \leftarrow \mathcal{E}_{2}$ are elementary equivalences of $n$-extensions of $A$ by $B$ in $\mathcal{A}_{3}$ with $A, B, \mathcal{E}$ in $\mathcal{A}_{1}$, then there are elementary equivalences

$$
\mathcal{E} \rightarrow \mathcal{E}_{1}^{\prime} \leftarrow \mathcal{E}_{2}^{\prime} \rightarrow \mathcal{E}_{2}
$$

with $\mathcal{E}_{1}^{\prime}, \mathcal{E}_{2}^{\prime}$ in $\mathcal{A}_{1}$ : using $(*)$, we may assume $\mathcal{E}_{2}=\mathcal{E}_{2}^{\prime}$ is in $\mathcal{A}_{1}$. Now choose $\mathcal{E}_{1}^{\prime} \hookrightarrow \mathcal{E}_{1}$ in $\mathcal{A}_{1}$ such that the components of $\mathcal{E}_{1}^{\prime}$ contain the image of the components of $\mathcal{E}$ and $\mathcal{E}_{2}^{\prime}$.

One proceeds similarly for $\mathcal{A}_{2} \hookrightarrow \mathcal{A}_{3}$.

q.e.d.

Corollary 1.8: For any $X \in \operatorname{Rep}_{F}(W)$,

$$
H^{\cdot}(W, X)=\underset{j}{\lim } H^{\cdot}\left(W_{j}, X\right) .
$$

\section{Proof:}

$$
\begin{aligned}
H^{\cdot}(W, X) & =\operatorname{Ext}_{\operatorname{Rep}_{F}(W)}(F, X) \\
& =\underset{\vec{j}}{\lim } \operatorname{Ext}_{\operatorname{Rep}_{F}\left(W_{j}\right)}(F, X) \\
& =\underset{\vec{j}}{\lim } H^{\cdot}\left(W_{j}, X\right) .
\end{aligned}
$$


Lemma 1.9: If $W$ is algebraic, then for any $X \in \operatorname{Rep}_{F}(W)$, the natural map

$$
H^{\cdot}(W, X) \longrightarrow H^{\cdot}(\mathfrak{w}, X)
$$

is an isomorphism. Note that the left hand side is calculated via injective resolutions in $\operatorname{Rat}(W)$, while the right hand side is calculated via injective resolutions in $\operatorname{Mod}_{\mathfrak{U}(\mathfrak{w})}$.

Proof: By filtering $X$, we may suppose $X=F$. By filtering $W$, we may suppose $W=\mathbb{G}_{a}$. Then the cohomology groups are well known and coincide. q.e.d.

It is possible to describe the category $R(W)^{\mathrm{opp}}$ explicitly as follows: let $T(W)$ be the category of topological $\hat{\mathfrak{U}}(\mathfrak{w})$-modules $M$ such that a) the topology is given by a countable descending filtration

$$
M=M^{0} \supset M^{1} \supset \ldots
$$

of $\hat{\mathfrak{U}}(\mathfrak{w})$-modules of finite codimension.

b) $M \stackrel{\sim}{\stackrel{\sim}{\longrightarrow}} \lim _{i} M / M^{i}$.

Then the two functors

$$
\begin{aligned}
& \stackrel{c}{\vee}: T(W)^{\text {opp }} \longrightarrow R(W), M \longmapsto \operatorname{Hom}_{F, \text { cont. }}(M, F), \\
& \vee: R(W)^{\text {opp }} \longrightarrow T(W), X \longmapsto \operatorname{Hom}_{F}(X, F)
\end{aligned}
$$

define an identification of $T(W)$ and $R(W)^{\text {opp }}$. Note that $\hat{\mathfrak{U}}(\mathfrak{w})$ itself lies in $T(W)$.

Dualizing 1.6.b) and its proof, one obtains

Corollary 1.10: (compare [H1], Theorem 3.4.)

$T(W)$ is an abelian category with surjective epimorphisms, injective monomorphisms and enough projectives. More precisely, for all $M \in T(1)$ the completed tensor product $\hat{\mathfrak{U}}(\mathfrak{w}) \hat{\otimes}_{F} M$ is projective in $T(W)$.

Proof: The functor ${ }^{\vee}$ transforms injective maps into surjective maps and vice versa.

q.e.d.

The category $\operatorname{Rep}_{F}(W)$ is contained in $T(W)$. Because of 1.6.b), we get for objects $X, Y \in \operatorname{Rep}_{F}(W)$ :

$\operatorname{Ext}_{\operatorname{Rep}_{F}(W)}(X, Y)=\operatorname{Ext}_{\operatorname{Rep}_{F}(W)}\left(Y^{\vee}, X^{\vee}\right)=\operatorname{Ext}_{R(W)}^{\cdot}\left(Y^{\vee}, X^{\vee}\right)=\operatorname{Ext}_{T(W)}^{.}(X, Y)$. 
In particular, by 1.6.c), for any $X \in \operatorname{Rep}_{F}(W)$, Hochschild cohomology $H^{\cdot}(W, X)$ coincides with $\operatorname{Ext}_{T(W)}(F, X)$. So if $L / F$ is a field extension, the natural map

$$
H^{\cdot}(W, X) \otimes_{F} L \longrightarrow H^{\cdot}\left(W_{L}, X \otimes_{F} L\right)
$$

is an isomorphism: apply 1.10 and use the fact that base change by $L$ is exact and maps $\hat{\mathfrak{U}}(\mathfrak{w})$ to $\hat{\mathfrak{U}}\left(\mathfrak{w}_{L}\right)$.

Now consider the right exact functor

$$
\Lambda: T(W) \rightarrow T(1), M \mapsto H_{0}(W, M)=M / \mathfrak{a} M=M \hat{\otimes}_{\hat{\mathfrak{U}(\mathfrak{w})}} F
$$

Using projective resolutions, first in $T(W)$ and then in $T(W) \times T(W)$, one shows that the left derivatives $H_{k}\left(W,{ }_{-}\right)$exist and can be calculated by resolving either $F$ or the module in question.

Lemma 1.11: If $W$ is algebraic, then for any $M \in \operatorname{Rep}_{F}(W)$, the natural map

$$
H .(W, M) \longrightarrow H .(\mathfrak{w}, M)
$$

is an isomorphism. Note that the right hand side is calculated via projective resolutions in $\operatorname{Mod}_{\mathfrak{U}(\mathfrak{w})}$.

Proof: as in 1.9 .

q.e.d.

\section{Proposition 1.12:}

a) Assume $H_{k}(W, F)$ is finite-dimensional for all $k \in \mathbb{N}_{0}$.

This is true in particular if $W$ is algebraic. Then

$$
H_{k}(W, M)=\lim _{i} H_{k}\left(W, M / M^{i}\right)
$$

for all $k \in \mathbb{N}_{0}$ and $M=\underset{\leftarrow}{\lim _{i}} M / M^{i} \in T(W)$.

b) Assume $H^{k}(W, F)$ is finite-dimensional for all $k \in \mathbb{N}_{0}$.

This is true in particular if $W$ is algebraic. Then

$$
H^{k}(W, M):={\underset{\iota}{i}}_{i} H^{k}\left(W, M / M^{i}\right)=\operatorname{Ext}_{T(W)}^{k}(F, M)
$$

for all $k \in \mathbb{N}_{0}$ and $M={\underset{\lim }{\leftarrow}}_{i} M / M^{i} \in T(W)$. 
Proof: By filtering the $M / M^{i}$, we get the finiteness condition for the $H_{k}\left(W, M / M^{i}\right)$ or $H^{k}\left(W, M / M^{i}\right)$ as well. The statement follows from a standard application of the Mittag-Leffler criterion.

Corollary 1.13: Let $W_{1} \leq W_{2}$ be algebraic unipotent groups, $d_{1}:=\operatorname{dim} W_{1}$. Then

$$
H^{k}\left(W_{1}, \hat{\mathfrak{U}}\left(\mathfrak{w}_{2}\right)\right)=\left\{\begin{array}{ll}
0, & k \neq d_{1} \\
H_{0}\left(W_{1}, \hat{\mathfrak{U}}\left(\mathfrak{w}_{2}\right)\right) \hat{\otimes}_{F} \Lambda^{d_{1}} \mathfrak{w}_{1}^{\vee}, & k=d_{1}
\end{array} .\right.
$$

Proof: By 1.9, 1.11, 1.12.a) and [K], Theorem 6.10, we have canonically

$$
H^{k}\left(W_{1}, \hat{\mathfrak{U}}\left(\mathfrak{w}_{2}\right)\right)=H_{d_{1}-k}\left(W_{1}, \hat{\mathfrak{U}}\left(\mathfrak{w}_{2}\right)\right) \hat{\otimes}_{F} \Lambda^{d_{1}} \mathfrak{w}_{1}^{\vee}
$$

So we have to show that $H_{n}\left(W_{1}, \hat{\mathfrak{U}}\left(\mathfrak{w}_{2}\right)\right)$ is zero unless $n=0$. Letting $d_{2}:=\operatorname{dim} W_{2}$, this will be achieved by induction, first on $d_{2}-d_{1}$ and then on $d_{1}$ :

If $d_{1}=d_{2}$, then $W_{1}=W_{2}$, and so $\hat{\mathfrak{U}}\left(\mathfrak{w}_{1}\right) \in T\left(W_{1}\right)$ is projective. If $d_{1}=0$, then the claim is trivial. In the general case, if $Z\left(W_{2}\right) \cap W_{1}$ is non-zero and unequal to $W_{1}$, we use the Hochschild-Serre spectral sequence together with the isomorphism

$$
H_{0}\left(Z\left(W_{2}\right) \cap W_{1}, \hat{\mathfrak{U}}\left(\mathfrak{w}_{2}\right)\right) \cong \hat{\mathfrak{U}}\left(\mathfrak{w}_{2} /\left(z\left(\mathfrak{w}_{2}\right) \cap \mathfrak{w}_{1}\right)\right) .
$$

If $Z\left(W_{2}\right) \cap W_{1}$ is zero, choose a one-dimensional subgroup $W^{\prime}$ of $Z\left(W_{2}\right)$. Then $W^{\prime} \times W_{1}$ is a subgroup of $W_{2}$ of dimension $d_{1}^{\prime}=d_{1}+1$. So $d_{2}-d_{1}^{\prime}=d_{2}-d_{1}-1$, and

$$
H_{m}\left(W^{\prime} \times W_{1}, \hat{\mathfrak{U}}\left(\mathfrak{w}_{2}\right)\right)=0 \text { unless } m=0 .
$$

Now use the Hochschild-Serre spectral sequence and the fact that $H_{p}\left(W^{\prime},{ }_{-}\right)$is zero for $p>1$ to conclude that

$$
H_{0}\left(W^{\prime}, H_{n}\left(W_{1}, \hat{\mathfrak{U}}\left(\mathfrak{w}_{2}\right)\right)\right)=0 \text { unless } n=0 .
$$

But any $M \in T\left(W^{\prime}\right)$ with trivial co-invariants is itself trivial.

It remains to consider the case where $W_{1} \leq Z\left(W_{2}\right)$. Clearly our claim follows if we manage to show that $\hat{\mathfrak{U}}\left(\mathfrak{w}_{2}\right) \in T\left(W_{1}\right)$ is projective. Choose a filtration

$$
0=\mathfrak{w}^{(k+1)} \subset \ldots \subset \mathfrak{w}^{(1)}=\mathfrak{w}_{2}
$$

of sub-Lie algebras satisfying $\left[\mathfrak{w}^{(i)}, \mathfrak{w}^{(j)}\right] \subset \mathfrak{w}^{(i+j)}$ and $\mathfrak{w}_{1}=\mathfrak{w}^{(k)}$. Choose a basis $\left(w_{1}, \ldots, w_{d_{1}}, w_{d_{1}+1}, \ldots\right)$ of $\mathfrak{w}_{2}$ respecting this filtration. 
By the Poincaré-Birkhoff-Witt theorem ([Hum2], 17.3, Corollary C),

$$
\left(w_{1}^{n_{1}} \cdot \ldots \cdot w_{d_{2}}^{n_{d_{2}}} \mid n_{1}, \ldots, n_{d_{2}} \in \mathbb{N}_{0}\right)
$$

is a basis of $\mathfrak{U}\left(\mathfrak{w}_{2}\right)$. Hence the $\mathfrak{a}$-adic filtration of $\hat{\mathfrak{U}}\left(\mathfrak{w}_{2}\right)$ is equivalent to the filtration $F$ by degree, where we set

$$
\operatorname{deg}\left(w_{j}\right):=\max \left\{m \mid w_{j} \in \mathfrak{w}^{(m)}\right\} .
$$

So another application of the Poincaré-Birkhoff-Witt theorem gives an isomorphism

$$
\hat{\mathfrak{U}}\left(\mathfrak{w}_{2}\right) \cong \hat{\mathfrak{U}}\left(\mathfrak{w}_{1}\right) \hat{\otimes}_{F} M
$$

of objects of $T\left(W_{1}\right)$, where $M \in T(1)$ is the completion with respect to the degree of the vector space with basis

$$
\left(w_{d_{1}+1}^{n_{d_{1}+1}} \cdot \ldots \cdot w_{d_{2}}^{n_{d_{2}}} \mid n_{d_{1}+1}, \ldots, n_{d_{2}} \in \mathbb{N}_{0}\right)
$$

Corollary 1.10 then concludes the proof.

q.e.d.

\section{$\S 2$ The generic pro-sheaf}

We begin this paragraph by recalling the notion of the pro-unipotent envelope of an abstract or profinite group.

We then use the results of $\S 1$ for a reinterpretation of Chen's construction of a pro-mixed Hodge structure on the completed group ring of the fundamental group of a smooth complex algebraic variety $X([\mathrm{C}]$; see also $[\mathrm{H} 2])$ together with the classification theorem for admissible unipotent variations of Hodge structure on $X$ ([HZ1], Theorem 1.6; see also [HZ2], §2) in Tannakian terms (Corollary 2.7). This result is a prototype of those that will follow in this and the next paragraph: the analogous statement for mixed lisse $\lambda$-adic sheaves is also true and follows from results in [SGA1]. The universal property 2.6 of what we call the generic pro-variation suffices to generate what amounts to a descent datum for the weight and Hodge filtration of the underlying vector bundle, thereby defining the de Rham version of the generic sheaf. We remark that for $X=\mathbb{P}^{1} \backslash\{0 ; 1 ; \infty\}$, the main results of this paragraph $(2.7,2.9,2.12)$ occur implicitly in $[\mathrm{B}]$ in the form: " $L(X)^{g}$ [which coincides with $\operatorname{Lie}\left(W_{\bar{x}}\right)$ in our notation] is the free Lie algebra in two variables" ([B], 1.3.1.ii)). 
So let $\pi$ be an abstract, finitely generated group,

$\mathbb{Q}[\pi]$ its group ring,

$\mathbb{Q}[\pi]^{\wedge}$ its completion with respect to the augmentation ideal $\mathfrak{a}$,

$W(\pi)$ the Tannaka dual of the category of unipotent representations of $\pi$ over

$\mathbb{Q}$, i.e., the pairs $(X, \rho)$, where $\operatorname{dim}_{\mathbb{Q}} X<\infty, \rho: \pi \rightarrow \mathrm{GL}(X)(\mathbb{Q})$ a group homomorphism such that $X$ has a filtration of $\pi$-submodules whose graded objects are trivial $\pi$-modules,

$\mathfrak{w}(\pi):=\operatorname{Lie}(W(\pi))$.

By $1.3, W(\pi)$ is pro-unipotent, and by the explicit construction of $W(\pi)$ (as reviewed in [D4], §9), it is a countable projective limit of unipotent groups, i.e., it satisfies the hypothesis set up before 1.6. Observe that $\operatorname{Rep}_{\mathbb{Q}}(W)$ is the category of finite-dimensional discrete $\mathbb{Q}[\pi]^{\wedge}$-modules.

Definition: $W(\pi)$ is called the pro-unipotent envelope of $\pi$.

$\mathfrak{w}(\pi)$ is called the Malcev-Lie algebra of $\pi$.

Example: Let $W / \mathbb{Q}$ be a unipotent algebraic group, $\pi \leq W(\mathbb{Q})$ an arithmetic subgroup.

By [D4], 9.5, $W$ is the pro-unipotent envelope of $\pi$.

Lemma 2.1: $\mathbb{Q}[\pi]^{\wedge}$ and $\hat{\mathfrak{U}}(\mathfrak{w}(\pi))$ are canonically isomorphic.

Proof: Both are objects of $T(W(\pi))$. Whenever an object of $T(W(\pi))$ together with an element is given, there exists a unique morphism of $\mathbb{Q}[\pi]^{\wedge}$ into this object sending 1 to the given element. $\hat{\mathfrak{U}}(\mathfrak{w}(\pi))$ has the same universal property.

q.e.d.

\section{Proposition 2.2:}

a) The functor

$$
\begin{aligned}
\text { \{finitely generated groups }\} & \longrightarrow\{\text { pro-unipotent groups }\} \\
\pi & \longmapsto W(\pi)
\end{aligned}
$$

is right exact.

b) For any field $F$ of characteristic $0, W(\pi) \hat{\otimes}_{\mathbb{Q}} F$ is the Tannaka dual of the category of unipotent representations of $\pi$ over $F$.

Proof: a) Let $\pi^{\prime} \stackrel{i}{\rightarrow} \pi \stackrel{p}{\rightarrow} \pi^{\prime \prime} \rightarrow 1$ be an exact sequence of finitely generated 
groups, i.e., $p$ surjective, $\operatorname{im}(i)=\operatorname{ker}(p)$,

$$
W^{\prime} \stackrel{i}{\longrightarrow} W \stackrel{p}{\longrightarrow} W^{\prime \prime} \longrightarrow 1
$$

the sequence of the Tannaka duals.

That $p$ is faithfully flat follows from 1.2.a).

Next, we obviously have $p \circ i=0$.

By 1.4.a), $\operatorname{im}(i)=\operatorname{ker}(p)$.

b) Let $W:=W(\pi)$. There is a natural map $\pi \rightarrow W(\mathbb{Q})$. It induces a functor

$$
\operatorname{Rep}_{F}(W) \longrightarrow\{\text { unipotent representations of } \pi \text { over } F\}
$$

Conversely, if $\pi \rightarrow \mathrm{GL}_{F}(X)$ is a unipotent representation, we get a map

$$
F[\pi]^{\wedge}=\hat{\mathfrak{U}}\left(\mathfrak{w} \hat{\otimes}_{\mathbb{Q}} F\right) \rightarrow \operatorname{End}_{F}(X)
$$

(by 2.1), i.e., a rational representation of $W$ over $F$.

Now let $\hat{\pi}$ be a profinite group, which is topologically finitely generated.

Let $l \in \mathbb{N}$ be a prime and define $W_{l}(\hat{\pi})$ as the Tannaka dual of the category of continuous unipotent representations of $\hat{\pi}$ over $\mathbb{Q}_{l}$, and let $\mathfrak{w}_{l}(\hat{\pi}):=\operatorname{Lie}\left(W_{l}(\hat{\pi})\right)$. Here, we consider the $l$-adic topology on finite-dimensional $\mathbb{Q}_{l}$-vector spaces. We shall frequently allow ourselves to refer to continuous representations on $\mathbb{Q}_{l}$-vector spaces simply as $l$-adic representations.

Statements analogous to 2.2 hold, where in b) we only consider finite extensions of $\mathbb{Q}_{l}$; in particular $W_{l}(\hat{\pi})$ is a quotient of $W_{l}\left(\hat{\pi}^{\prime}\right)$ for a group $\hat{\pi}^{\prime}$, which is the profinite completion of some finitely generated group. By the next proposition, $W_{l}(\hat{\pi})$ also satisfies the hypothesis set up before 1.6.

Proposition 2.3: Let $\pi$ be a finitely generated group, $\hat{\pi}$ its profinite completion, $l \in \mathbb{N}$ prime, $F / \mathbb{Q}_{l}$ a finite extension.

a) $\operatorname{res}_{\hat{\pi}}^{\pi}$ induces an equivalence of categories

$\{$ continuous unipotent representations of $\hat{\pi}$ over $F\} \stackrel{\sim}{\longrightarrow}$

$\stackrel{\sim}{\longrightarrow}\{$ unipotent representations of $\pi$ over $F\}$.

b) $W_{l}(\hat{\pi})=W(\pi) \hat{\otimes}_{\mathbb{Q}} \mathbb{Q}_{l}$.

Proof: a) Any unipotent representation of $\pi$ over $F$ stabilizes some lattice over $o_{F}$, hence is continuous with respect to the profinite topology on $\pi$.

b) follows from a) and 2.2.b).

q.e.d. 
Definition: A finitely generated group $\pi$ is called pseudo-nilpotent, if

$$
H^{\cdot}(W(\pi), X) \longrightarrow H^{\cdot}(\pi, X)
$$

is an isomorphism for $X=\mathbb{Q}$.

This is then automatically true for all $X \in \operatorname{Rep}_{\mathbb{Q}}(W(\pi)) . H^{\cdot}(\pi, X)$ is ordinary group cohomology, calculated via injective resolutions in $\operatorname{Mod}_{\mathbb{Q}[\pi]}$.

Remarks: a) As in 1.9, one shows using the construction of $W(\pi)$ for nilpotent groups (compare [D4], §9) and the usual filtration argument, that nilpotent finitely generated groups are pseudo-nilpotent.

b) As 1.8 shows, this definition coincides with the one given in [H1], 5.3. More precisely, continuous cohomology of the fundamental group is the same as Hochschild cohomology of its pro-unipotent envelope.

This viewpoint can be employed e.g. for a simplification of the proof of the following result, which however won't be needed in the sequel.

Lemma 2.4: (compare [H1], Theorem 5.1.)

Let $\pi$ be a finitely generated group. Then the natural maps

$$
H^{k}(W(\pi), \mathbb{Q}) \rightarrow H^{k}(\pi, \mathbb{Q})
$$

are isomorphisms for $k=0,1$, and injective for $k=2$.

Proof: We interpret the cohomology groups as Yoneda-Ext groups in the category $\operatorname{Rep}_{\mathbb{Q}}(W(\pi))=\{$ unipotent representations of $\pi$ over $\mathbb{Q}$ \} as we may by 1.6.c), and the category $\operatorname{Mod}_{\mathbb{Q}[\pi]}$ of arbitrary $\pi$-modules respectively.

The claim for $k=0$ and $k=1$ is trivial.

So take a two-extension

$$
0 \rightarrow \mathbb{Q} \rightarrow E_{1} \rightarrow E_{2} \rightarrow \mathbb{Q} \rightarrow 0
$$

with unipotent representations $E_{1}$ and $E_{2}$ of $\pi$ over $\mathbb{Q}$, that becomes trivial in $\operatorname{Ext}_{\text {Mod } \mathbb{Q}[\pi]}^{2}(\mathbb{Q}, \mathbb{Q})$. This means precisely that there is a $\pi$-module $E$ together with a filtration

$$
0 \subset \mathbb{Q} \subset E_{1} \subset E
$$

by $\pi$-submodules such that $E / \mathbb{Q}=E_{2}$.

Thus $E$ is necessarily of finite dimension, and since it has a filtration with unipotent graded objects, it is itself unipotent.

q.e.d. 
We won't define quasi-nilpotency for profinite topologically finitely generated groups. If a quasi-nilpotent abstract group is the fundamental group of a topological space $\bar{X}$, and $\bar{X}$ is a $K(\pi, 1)$, then the cohomology of $\bar{X}$ can be calculated in the Tannaka category of unipotent representations of $\pi$. It is in this property that we shall be interested. We shall formulate it also in the $l$-adic context (compare $\S 4$ ).

For a nice overview concerning admissible graded-polarizable unipotent variations of mixed Hodge structure, see [HZ2], $\S \S 1-2$.

Let $X$ be a smooth complex variety. Assume $X$ is connected. Fix $x \in X(\mathbb{C})$ and write $\bar{X}:=X(\mathbb{C}), \bar{x}:=x$. There is a canonical mixed graded-polarizable $\mathbb{Q}$ Hodge structure $(\mathbb{Q}-M H S)$ on the truncated group ring $\mathbb{Q}\left[\pi_{1}(\bar{X}, \bar{x})\right] / \mathfrak{a}^{n}, n \in \mathbb{N}$ $([\mathrm{C}],[\mathrm{H} 2])$.

Thus, if we let $G$ denote the Tannaka dual of the category $M H_{\mathbb{Q}}$ of gradedpolarizable $\mathbb{Q}-M H S$ with respect to the forgetful functor

$$
M H_{\mathbb{Q}} \rightarrow \operatorname{Vec}_{\mathbb{Q}},
$$

we get a pro-algebraic action of $G$ on

$$
{\underset{\lim }{n}}_{\mathbb{n}} \mathbb{Q}\left[\pi_{1}(\bar{X}, \bar{x})\right] / \mathfrak{a}^{n}=\mathbb{Q}\left[\pi_{1}(\bar{X}, \bar{x})\right]^{\wedge} \underset{2.1}{=} \hat{\mathfrak{U}}\left(\mathfrak{w}\left(\pi_{1}(\bar{X}, \bar{x})\right)\right)=: \hat{\mathfrak{U}}_{\bar{x}},
$$

where, as before, $\mathfrak{w}\left(\pi_{1}(\bar{X}, \bar{x})\right)$ is the Malcev Lie-algebra of the finitely generated group $\pi_{1}(\bar{X}, \bar{x})$.

Multiplication within $\hat{\mathfrak{U}}_{\bar{x}}$ is a morphism of $M H S$, and the unit 1 is an element of $\left(W_{0, \mathbb{Q}} \cap F^{0}\right)\left(\hat{\mathfrak{U}}_{\bar{x}}\right)$.

Now let $\mathbf{V}$ be an admissible graded-polarizable unipotent variation of $\mathbb{Q}-M H S$ on $X$ and let

$$
\rho_{\bar{x}}: \hat{\mathfrak{U}}_{\bar{x}} \longrightarrow \operatorname{End}_{\mathbb{Q}} \mathbf{V}_{\bar{x}}
$$

be the monodromy representation.

Both sides carry pro- $\mathbb{Q}-M H S$, and we have

Theorem 2.5: ([HZ1], Theorem 7.2 or [HZ2], Theorem 2.2.)

$\rho_{\bar{x}}$ is a morphism of pro- $M H S$. 
Theorem 2.6: ([HZ1], Theorem 1.6 or [HZ2], Theorem 2.6.)

The functor $\mathbf{V} \mapsto \rho_{\bar{x}}$ is an equivalence of categories

$$
\left\{\begin{array}{l}
\text { admissible graded-- } \\
\text { polarizable uni- } \\
\text { potent variations } \\
\text { of } \mathbb{Q}-M H S \text { on } X
\end{array}\right\} \longrightarrow\left\{\begin{array}{l}
\text { graded-polarizable } \\
\mathbb{Q}-M H S \mathbf{H} \text { together with } \\
\text { a morphism of } \mathbb{Q}-M H S \\
\hat{\mathfrak{U}}_{\bar{x}} \longrightarrow \underline{\text { End }}(\mathbf{H}) \text { respecting } \\
\text { the algebra structure }
\end{array}\right\} .
$$

Let $P_{\bar{x}}$ be the Tannaka dual of the category $U \operatorname{Var}_{\mathbb{Q}}(X)$ of admissible gradedpolarizable unipotent variations of $\mathbb{Q}-M H S$ on $X$ with respect to the functor

$$
\text { "fibre at } \bar{x} ": U \operatorname{Var}_{\mathbb{Q}}(X) \longrightarrow \operatorname{Vec}_{\mathbb{Q}} \text {. }
$$

We have natural morphisms

$$
P_{\bar{x}} \underset{x}{\stackrel{\pi}{\rightleftarrows}} G
$$

belonging to

$$
\begin{aligned}
& x^{*}:=\text { "fibre at } x ", \\
& \pi^{*}:=\text { "associated constant variation" }
\end{aligned}
$$

Clearly $\pi \circ x=\operatorname{id}_{G}$, and by 1.3 ,

$$
W_{\bar{x}}:=\operatorname{ker}(\pi)
$$

is pro-unipotent. The tensor functor "monodromy representation" induces a morphism

$$
W\left(\pi_{1}(\bar{X}, \bar{x})\right) \longrightarrow P_{\bar{x}}
$$

which factors over $W_{\bar{x}}$.

Corollary 2.7: The natural morphism

$$
W\left(\pi_{1}(\bar{X}, \bar{x})\right) \stackrel{\eta_{\bar{x}}}{\longrightarrow} W_{\bar{x}}
$$

is an isomorphism.

Proof: We have to check the criteria of 1.4:

a) Let $\mathbf{V} \in U \operatorname{Var}_{\mathbb{Q}}(X)$. Then $\mathbf{V}_{\bar{x}}^{W\left(\pi_{1}(\bar{X}, \bar{x})\right)}$ is a sub-MHS of $\mathbf{V}_{\bar{x}}$ : observe that we have $\mathfrak{a}=W_{-1}\left(\hat{\mathfrak{U}}_{\bar{x}}\right)$ as follows from the explicit construction of the $M H S$ on $\hat{\mathfrak{U}}_{\bar{x}}$. (See [H2], Remark 5.4.) By 2.5, we have a morphism of $M H S$

$$
\mathbf{V}_{\bar{x}} \rightarrow \operatorname{Hom}_{\mathbb{Q}}\left(\mathfrak{a}, \mathbf{V}_{\bar{x}}\right), v \mapsto\left(\alpha \mapsto \rho_{\bar{x}}(\alpha) \cdot v\right) .
$$


Now $\mathbf{V}_{\bar{x}}^{W\left(\pi_{1}(\bar{X}, \bar{x})\right)}$ is the kernel of this morphism, i.e., it is a sub-MHS of $\mathbf{V}_{\bar{x}}$. It carries the trivial representation of $\hat{\mathfrak{U}}_{\bar{x}}$. By 2.6, it corresponds to a subobject of $\mathbf{V}$, which is clearly the largest subvariation, that is constant.

b) Multiplication within $\hat{\mathfrak{U}}_{\bar{x}}$ yields representations

$$
\hat{\mathfrak{U}}_{\bar{x}} \rightarrow \operatorname{End}_{\mathbb{Q}}\left(\hat{\mathfrak{U}}_{\bar{x}} / \mathfrak{a}^{n}\right), n \in \mathbb{N},
$$

which, as remarked earlier, are morphisms of MHS. 2.6 gives the desired action of $P_{\bar{x}}$ on $\hat{\mathfrak{U}}_{\bar{x}}$. $\quad$ q.e.d.

Remark: $\hat{\mathfrak{U}}_{\bar{x}}$, together with the action of $P_{\bar{x}}$ of the proof of 2.7 , defines a pro-object of $U \operatorname{Var}_{\mathbb{Q}}(X)$. This is not the tautological variation of [HZ2], $\S 1$. The latter corresponds to the adjoint action of $P_{\bar{x}}$ on $\hat{\mathfrak{U}}_{\bar{x}}$ (compare [D1], I, Proposition 1.6) and is independent of $x$. Its fibre at $\bar{y} \in \bar{X}$ is $\mathbb{Q}\left[\pi_{1}(\bar{X}, \bar{y})\right]^{\wedge}=\hat{\mathfrak{U}}_{\bar{y}}$, while the fibre of the above representation is $\mathbb{Q}\left[\Omega \frac{0}{x}, \bar{y}\right]^{\wedge}$ together with its natural $\hat{\mathfrak{U}}_{\bar{x}}$-module structure. Here, $\Omega_{\bar{x}}^{0}, \bar{y}$ denotes the set of homotopy classes of paths connecting $\bar{x}$ and $\bar{y}$. This is the canonical variation with base point $x$ of [HZ1], $\S 1$.

We suggest another terminology:

Definition: The pro-object of $U \operatorname{Var}_{\mathbb{Q}}(X)$ corresponding to $\hat{\mathfrak{U}}_{\bar{x}}$ with the mixed Hodge-representation given by left multiplication is called $\mathcal{G} e n_{x}$, the generic pro-unipotent variation with basepoint $x$ on $X$.

This terminology can be justified as follows: the finite-dimensional subquotients of $\mathcal{G} e n_{x}$, together with the constant variations, generate $U \operatorname{Var}_{\mathbb{Q}}(X)$ as a full Tannakian subcategory, that is closed under formation of subobjects. (Proof: by 1.2.a), the Tannaka dual of the subcategory is a quotient of $P_{\bar{x}}=W_{\bar{x}} \rtimes G$. But $G$ is still a quotient, and $W_{\bar{x}}$ still injects.)

The dependence of $\mathcal{G} e n_{x}$ on the basepoint is as follows: any path connecting $\bar{x}$ and $\bar{x}^{\prime}$ or, more generally, any isomorphism of fibre functors

$$
\text { "fibre at } \bar{x} \stackrel{\sim}{\longrightarrow} \text { "fibre at } \bar{x}^{\prime} "
$$

on $U \operatorname{Var}_{\mathbb{Q}}(X)$ defines an isomorphism $\mathcal{G}_{e x_{x^{\prime}}} \stackrel{\sim}{\longrightarrow} \mathcal{G} e n_{x}$.

Note that if we apply 1.5 to the result on the shape of $P_{\bar{x}}$ in 2.7 , we get back 2.6.

Statements 2.5-2.7 remain correct, possibly after applying $\otimes_{\mathbb{Q}} F$, if we replace $\mathbb{Q}$ by an arbitrary coefficient field $F$ contained in $\mathbb{R}$. 
Now for the $\lambda$-adic situation: fix a prime number $l$; let $F / \mathbb{Q}_{l}$ be finite, $\lambda \in F$ a prime element. Let $X$ be a quasi-compact scheme over a number field $k$, and assume that $\bar{X}:=X \otimes_{k} \bar{k}$ is connected and that $X(k) \neq \emptyset$. Let $x \in X(k)$. By [SGA1], Exp. IX, Théorème 6.1, there is an exact sequence of algebraic fundamental groups

$$
1 \longrightarrow \pi_{1}(\bar{X}, \bar{x}) \longrightarrow \pi_{1}(X, \bar{x}) \stackrel{\pi}{\longrightarrow} G_{k} \longrightarrow 1
$$

and the point $x$ gives a splitting of $\pi$. Here, $G_{k}:=\operatorname{Gal}(\bar{k} / k)$. Via this splitting, we write $\pi_{1}(X, \bar{x})=\pi_{1}(\bar{X}, \bar{x}) \rtimes G_{k}$.

This already resembles the Hodge-theoretic situation to a large extent. In fact, it is easy to see that the category of $\lambda$-adic representations of $\pi_{1}(X, \bar{x})$ is equivalent to the category of Galois-equivariant $\lambda$-adic representations of $\pi_{1}(\bar{X}, \bar{x})$. However, this is not quite what we want. We need to consider $\lambda$-adic sheaves on $X$, that are mixed in the sense of [D3], VI:

Definition: Let $F$ be a finite extension of $\mathbb{Q}_{l}$, and let $X / k$ be separated and of finite type.

i) $\mathrm{Et}_{F}^{l, m}(X)$ is the full subcategory of the category of constructible $F$-sheaves on $X$ consisting of objects $\mathbf{V}$ satisfying

a) $\mathbf{V}$ is lisse.

b) There is a finite set $S \subset \operatorname{Spec}\left(o_{k}\right)$ containing the primes dividing $l$, a separated scheme $\mathfrak{X} \rightarrow \operatorname{Spec}\left(o_{S}\right)$ of finite type and a lisse constructible $F$-sheaf $\mathfrak{V}$ on $\mathfrak{X}$ such that

a) $X=\mathfrak{X} \otimes_{o_{S}} k$, $\mathbf{V}=\mathfrak{V} \otimes_{o_{S}} k$.

$\beta) \mathfrak{V}$ is mixed in the sense of [D3], Définition 1.2.2.

In particular, there is a weight filtration $W$. of $\mathfrak{V}$.

ii) $U \mathrm{Et}_{F}^{l, m}(X)$ is the full subcategory of those $\mathbf{V} \in \mathrm{Et}_{F}^{l, m}(X)$ admitting a filtration, whose graded objects are geometrically trivial.

It is not difficult to see that if $X$ is smooth and if such a filtration exists, the weight filtration will have the same property. (Use [D3], Théorème 6.1.2, generic base change ([SGA4 1/2], Th. finitude, Théorème 1.9) and [D3], Corollaire 3.3.5 to see that a pure sheaf in $U \mathrm{Et}_{F}^{l, m}(X)$ is geometrically trivial.) 
In terms of continuous representations, property i)b) implies that

$$
\pi_{1}(X, \bar{x}) \longrightarrow \mathrm{GL}_{F}\left(\mathbf{V}_{\bar{x}}\right)
$$

factors through $\pi_{1}(\mathfrak{X}, \bar{x})$ for a suitable extension $\mathfrak{X}$ of $X$.

Property ii) means precisely that the induced representation of $\pi_{1}(\bar{X}, \bar{x})$ is unipotent. It is not hard to see that $U_{\mathrm{Et}_{F}^{l, m}}^{l, X}$ is a neutral Tannakian category over $F$.

We let $\pi$ denote the morphism

$$
X \longrightarrow \operatorname{Spec}(k) .
$$

By functoriality of the Malcev Lie algebra, the point $x$ defines a continuous action of $G_{k}$ on

$$
\hat{\mathfrak{U}}\left(\mathfrak{w}_{l}\left(\pi_{1}(\bar{X}, \bar{x})\right)\right) \otimes_{\mathbb{Q}_{l}} F=: \hat{\mathfrak{U}}_{\bar{x}} .
$$

Note that by the comparison theorem ([SGA1], Exp. XII, Corollaire 5.2), $\pi_{1}(\bar{X}, \bar{x})$ is topologically finitely generated.

As in the Hodge-theoretic setting, the first thing we have to make sure is that $\hat{\mathfrak{U}}_{\bar{x}}$ itself belongs to pro- $\operatorname{Et}_{F}^{l, m}(\operatorname{Spec}(k))$ :

Theorem 2.8: Assume $X$ is smooth.

Then the representation of $G_{k}$ on $\hat{\mathfrak{U}}_{\bar{x}}$ is mixed.

Proof: $\operatorname{Et}_{F}^{l, m}(\operatorname{Spec}(k))$ is closed under formation of quotients in the category of constructible $F$-sheaves on $\operatorname{Spec}(k)$ ([J], Lemma 6.8.1.b)). Take an affine neighbourhood $U$ of $x$. As $\bar{X}$ is normal and $\bar{U}$ is dense in $\bar{X}, \pi_{1}(\bar{X}, \bar{x})$ is a quotient of $\pi_{1}(\bar{U}, \bar{x})$. So we may assume $X$ is affine.

By [Hi], Main Theorem I and Corollary 3 of Main Theorem II, $X$ can be embedded as an open dense subvariety in a smooth projective variety $Z / k$ such that $Z \backslash X$ is a divisor with normal crossings.

There is a finite set $S \subset \operatorname{Spec}\left(o_{k}\right)$ containing the primes dividing $l$ such that there is a smooth extension

$$
\mathfrak{X} \stackrel{\varpi}{\longrightarrow} \operatorname{Spec}\left(o_{S}\right)
$$

of $X$ with geometrically connected fibres, which is the complement of a relative divisor with normal crossings in a smooth, projective scheme over $\operatorname{Spec}\left(o_{S}\right)$ and such that $x$ extends to a section

$$
\operatorname{Spec}\left(o_{S}\right) \stackrel{\mathcal{X}}{\longrightarrow} \mathfrak{X} .
$$


Now we may apply [SGA1], Exp. XIII, Proposition 4.3 and Exemples 4.4 to conclude that the natural sequence

$$
1 \longrightarrow \pi_{1}^{l}(\bar{X}, \bar{x}) \longrightarrow \pi_{1}^{\prime}(\mathfrak{X}, \bar{x}) \underset{\mathcal{X}}{\stackrel{\varpi}{\rightleftarrows}} \pi_{1}\left(\operatorname{Spec}\left(o_{S}\right), \overline{\pi(x)}\right) \longrightarrow 1
$$

is split exact.

Here, $\pi_{1}^{l}(\bar{X}, \bar{x})$ is the largest pro-l quotient of $\pi_{1}(\bar{X}, \bar{x})$, and $\pi_{1}^{\prime}(\mathfrak{X}, \bar{x})$ is the quotient of $\pi_{1}(\mathfrak{X}, \bar{x})$ by $N$, where $\operatorname{ker}(\varpi) / N$ is the largest pro- $l$ quotient of $\operatorname{ker}(\varpi)$. So the action of $G_{k}$ on $\pi_{1}^{l}(\bar{X}, \bar{x})$ factors through $\pi_{1}\left(\operatorname{Spec}\left(o_{S}\right), \overline{\pi(x)}\right)$.

Now observe that

$$
\mathfrak{w}_{l}\left(\pi_{1}(\bar{X}, \bar{x})\right)=\mathfrak{w}_{l}\left(\pi_{1}^{l}(\bar{X}, \bar{x})\right)
$$

since every unipotent $\lambda$-adic representation of $\pi_{1}(\bar{X}, \bar{x})$ factors through $\pi_{1}^{l}(\bar{X}, \bar{x})$. So the representation of $G_{k}$ on $\hat{\mathfrak{U}}_{\bar{x}}$ factors through $\pi_{1}\left(\operatorname{Spec}\left(o_{S}\right), \overline{\pi(x)}\right)$.

We now define the weight filtration on $\hat{\mathfrak{U}}_{\bar{x}}$. It will automatically be compatible with multiplication on $\hat{\mathfrak{U}}_{\bar{x}}$.

Let $\mathfrak{a}$ denote the augmentation ideal. Set $W_{0} \hat{\mathfrak{U}}_{\bar{x}}:=\hat{\mathfrak{U}}_{\bar{x}}, W_{-1} \hat{\mathfrak{U}}_{\bar{x}}:=\mathfrak{a}$.

Now observe that $\mathfrak{a} / \mathfrak{a}^{2} \stackrel{\sim}{\longrightarrow} H^{1}(\bar{X}, F(0))^{\vee}=\left(R^{1} \varpi_{*} F_{\mathfrak{X}}(0)\right)_{\text {gen.pt. }}^{\vee}$, which is mixed of weights -1 and -2 . Writing

$$
p: \mathfrak{a} \longrightarrow \mathfrak{a} / \mathfrak{a}^{2},
$$

we set $W_{-2} \hat{\mathfrak{U}}_{\bar{x}}:=p^{-1}\left(W_{-2}\left(\mathfrak{a} / \mathfrak{a}^{2}\right)\right)$.

Now let $W$. be the filtration "generated" by $W_{-1}$ and $W_{-2}$.

More explicitly, $\mathfrak{a}^{n} \subset W_{-n} \hat{\mathfrak{U}}_{\bar{x}}$ for all $n \in \mathbb{N}_{0}$, and $W_{-n}\left(\hat{\mathfrak{U}}_{\bar{x}} / \mathfrak{a}^{n}\right)$ is spanned by products of the form $\alpha_{1} \cdot \ldots \cdot \alpha_{m} \cdot \beta_{1} \cdot \ldots \cdot \beta_{l}$, where $\alpha_{i} \in W_{-1} \hat{\mathfrak{U}}_{\bar{x}}, \beta_{j} \in W_{-2} \hat{\mathfrak{U}}_{\bar{x}}$ and $m+2 l \geq n$. With this definition, the canonical surjection

$$
H^{1}(\bar{X}, F(0))^{\otimes(-n)} \longrightarrow \mathfrak{a}^{n} / \mathfrak{a}^{n+1}
$$

respects the weight filtration for all $n \in \mathbb{N}_{0}$. It follows that the graded parts are pure.

q.e.d.

As before, let $X / k$ be a smooth, separated, geometrically connected scheme of finite type, $x \in X(k)$. Define a lisse pro- $F$-sheaf $\mathcal{G} e n_{x}$ on $X$, i.e., a continuous representation of $\pi_{1}(X, \bar{x})=\pi_{1}(\bar{X}, \bar{x}) \rtimes G_{k}$ as follows:

the underlying vector space is $\hat{\mathfrak{U}}_{\bar{x}}, \pi_{1}(\bar{X}, \bar{x})$ acts by multiplication, and $G_{k}$ acts as in 2.8. By construction, the induced representation of $\pi_{1}(\bar{X}, \bar{x})$ is prounipotent, and the filtration $W$. on $\hat{\mathfrak{U}}_{\bar{x}}$ defines a filtration $W \cdot \mathcal{G} e n_{x}$ by lisse subpro-sheaves. 
Using the same techniques as in the proof of 2.8 , it follows that $\mathcal{G} e n_{x}$ is an object of pro- $U \mathrm{Et}_{F}^{l, m}(X)$ if $X$ is quasi-projective, the graded objects being sums of quotients of $\pi^{*}\left(H^{1}(\bar{X}, F(0))\right)^{\otimes(-n)}, n \in \mathbb{N}_{0}$. In order to obtain the statement for general $X$, we have to study the dependence of $\mathcal{G} e n_{x}$ on $x$ :

let $x^{\prime} \in X(k)$ be another point, and denote by $\zeta$ the generic point of $X$.

Choose specialization maps $\bar{\zeta} \rightarrow \bar{x}, \bar{\zeta} \rightarrow \bar{x}^{\prime}$ (compare [SGA4,II], Exp. VIII, 7.2).

These give an isomorphism of fibre functors

$$
\text { "fibre at } \bar{x} \stackrel{\sim}{\longrightarrow} \text { "fibre at } \bar{x}^{\prime \prime} \quad \text { on } U \operatorname{Et}_{F}^{l}(X) \text {, }
$$

the category of unipotent lisse $F$-sheaves on $X$.

Any such isomorphism induces an isomorphism

$$
\tilde{P}_{\bar{x}^{\prime}} \stackrel{\sim}{\longrightarrow} \tilde{P}_{\bar{x}}
$$

of the corresponding Tannaka duals, which in particular yields an isomorphism

$$
\mathcal{G}_{e n_{x^{\prime}}} \stackrel{\sim}{\longrightarrow} \mathcal{G} e n_{x},
$$

$W_{l}\left(\pi_{1}(\bar{X}, \bar{x})\right) \otimes_{\mathbb{Q}_{l}} F$ and $W_{l}\left(\pi_{1}\left(\bar{X}, \bar{x}^{\prime}\right)\right) \otimes_{\mathbb{Q}_{l}} F$ being the respective kernels of

$$
\tilde{P}_{\bar{x}} \longrightarrow \tilde{G}, \tilde{P}_{\bar{x}^{\prime}} \longrightarrow \tilde{G},
$$

where $\tilde{G}$ is the Tannaka dual of $\operatorname{Et}_{F}^{l}(\operatorname{Spec}(k))$.

Theorem 2.9: Let $X$ be a smooth, separated, geometrically connected scheme of finite type over a number field $k$, and let $x \in X(k)$.

a) $\mathcal{G} e n_{x}$ is an object of pro- $U \operatorname{Et}_{F}^{l, m}(X)$.

b) Let $P_{\bar{x}}$ be the Tannaka dual of $U \operatorname{Et}_{F}^{l, m}(X)$ with respect to

$$
\text { "fibre at } \bar{x} ": U \mathrm{Et}_{F}^{l, m}(X) \longrightarrow \operatorname{Vec}_{F},
$$

and let

$$
P_{\bar{x}} \underset{x}{\stackrel{\pi}{\rightleftarrows}} G
$$

be the morphisms induced by $\pi^{*}$ and "fibre at $x$ ", where $G$ is the Tannaka dual of $\operatorname{Et}_{F}^{l, m}(\operatorname{Spec}(k))$. Then the natural morphism

$$
W_{l}\left(\pi_{1}(\bar{X}, \bar{x})\right) \otimes_{\mathbb{Q}_{l}} F \stackrel{\eta_{\bar{x}}}{\longrightarrow} W_{\bar{x}}:=\operatorname{ker}(\pi)
$$

is an isomorphism. 
c) The functor

$$
\begin{aligned}
& U \mathrm{Et}_{F}^{l, m}(X) \longrightarrow\left\{\begin{array}{l}
\text { objects } \mathbf{H} \text { of } \mathrm{Et}_{F}^{l, m}(\operatorname{Spec}(k)) \\
\text { together with a morphism } \\
\text { of Galois modules } \\
\hat{\mathfrak{U}}_{\bar{x}} \longrightarrow \underline{\operatorname{End}}(\mathbf{H}) \text { respecting } \\
\text { the algebra structure }
\end{array}\right\}, \\
& \mathbf{V} \longmapsto \mathbf{V}_{\bar{x}}
\end{aligned}
$$

is an equivalence of categories.

Proof: We prove a), leaving b) and c) to the reader. (Hint: use 1.4 and 1.5!) Let $X=\bigcup_{i=1}^{n} U_{i}$ be an affine covering. Using [EGAIV,3], Théorème 8.8.2 and the unicity of the weight filtration of a mixed sheaf, it is not hard to see that we may replace $k$ by a finite extension. So we may assume that every $U_{i}$ contains a $k$-rational point $x_{i}$.

As remarked before, $\mathcal{G} e n_{U_{i}, x_{i}} \in U \operatorname{Et}_{F}^{l, m}\left(U_{i}\right)$. But $\mathcal{G} e n_{x}$ is isomorphic to $\mathcal{G} e n_{x_{i}}$, whose restriction to $U_{i}$ is a quotient of $\mathcal{G} e n_{U_{i}, x_{i}}$. So $\left.\mathcal{G} e n_{x}\right|_{U_{i}}$ is mixed for all $i$. Again using [EGAIV,3], Théorème 8.8.2, one sees that after possibly enlarging $S$, the extensions of $U_{i}$ to $\operatorname{Spec}\left(o_{S}\right)$ glue together to give an extension $\mathfrak{X}$ of $X$. The extensions of $\mathcal{G} e n_{x \mid U_{i}}$, being lisse and coinciding on a non-empty open subset of $X$, necessarily glue together to form an extension of $\mathcal{G e n}$, which is mixed.

2.9 remains correct if we consider $\overline{\mathbb{Q}_{l}}$-sheaves instead of $F$-sheaves.

Remark: If one replaces $U \operatorname{Var}_{F}$ or $U E t_{F}^{l, m}$ by a full Tannakian subcategory $\mathcal{C}$ closed under formation of subobjects, then 2.7 and 2.9.b) will be false in general.

However, as the proofs show, we obtain a correct statement once we replace $W_{(l)}\left(\pi_{1}(\bar{X}, \bar{x})\right) \hat{\otimes}_{\mathbb{Q}_{(l)}} F$ by its largest quotient $W_{\mathcal{C}, \bar{x}}$ such that

$$
\operatorname{ker}\left(W_{(l)}\left(\pi_{1}(\bar{X}, \bar{x})\right) \hat{\otimes}_{\mathbb{Q}_{(l)}} F \rightarrow W_{\mathcal{C}, \bar{x}}\right)
$$

is normal in $P_{\bar{x}}$ and the corresponding quotient of $\mathcal{G} e n_{x}$ is contained in pro$\mathcal{C}(X){ }^{\dagger}$

For example, we could take $\mathcal{C}$ to be the category of objects whose graded parts

\footnotetext{
${ }^{\dagger}$ By definition, the subscript $(l)$ can take the two values blank and $l$, depending on whether one considers the Hodge or $l$-adic setting.
} 
are of Tate type.

Then

$$
W_{\mathcal{C}, \bar{x}}=\operatorname{ker}\left(P_{\mathcal{C}, \bar{x}} \stackrel{\pi}{\longrightarrow} G_{\mathcal{C}}\right)
$$

is the largest pro-Tate quotient of $W_{(l)}\left(\pi_{1}(\bar{X}, \bar{x})\right) \hat{\otimes}_{\mathbb{Q}_{(l)}} F$. It coincides with $W_{(l)}\left(\pi_{1}(\bar{X}, \bar{x})\right) \hat{\otimes}_{\mathbb{Q}_{(l)}} F$ if and only if $H^{1}\left(\bar{X}, \mathbb{Q}_{(l)}(0)\right)$ is of Tate type (compare the proof of 2.8), e.g. if $X=\mathbb{P}^{1} \backslash\left\{x_{1}, \ldots, x_{n}\right\}$.

Another possibility is to let $\mathcal{C}$ be the category of objects "of geometric origin". It seems to be reasonable to expect $\mathcal{G} e n_{x}$ to be of geometric origin, but I have no proof. But see the remark preceding Theorem 2.12.

As a first approximation to the definition of a category of smooth mixed motivic sheaves, we might let ourselves be inspired by Jannsen's or Deligne's definition of mixed realizations $([\mathrm{J}], \S 2,[\mathrm{D} 4], \S 1)$ :

\section{Definition:}

i) Let $k$ be a number field, $X / k$ smooth, separated and of finite type. $M S_{\mathbb{Q}}^{s}(X)$, the category of mixed systems of smooth sheaves on $X$ consists of families

$$
\left(\mathbf{V}_{l}, \mathbf{V}_{D R}, \mathbf{V}_{\infty, \sigma}, I_{l, \bar{\sigma}}, I_{D R, \sigma}, I_{\infty, \sigma} \mid l \in \mathbb{N} \text { prime, } \sigma: k \hookrightarrow \mathbb{C}, \bar{\sigma}: \bar{k} \hookrightarrow \mathbb{C}\right)
$$

where

a) $\mathbf{V}_{l} \in \operatorname{Et}_{\mathbb{Q}_{l}}^{l, m}(X)$

b) $\mathbf{V}_{D R}$ is a vector bundle on $X$, equipped with a flat connection $\nabla$, which is regular at infinity in the sense of [D1], II, remark following Définition 4.5. Further parts of the data are an ascending weight filtration $W$. by flat subbundles and a descending Hodge filtration $\mathcal{F}$. by subbundles.

c) $\mathbf{V}_{\infty, \sigma}$ is a variation of $\mathbb{Q}-M H S$ on $X_{\sigma}(\mathbb{C})$, which is admissible in the sense of $[\mathrm{Ka}]$. More precisely, in the notation established there, we require $\mathbf{V}_{\infty, \sigma}$ to be admissible with respect to any compactification of $X_{\sigma}$. By the proof of [Ka], Proposition 1.10.1, this definition does not depend on the choice of this compactification.

The underlying local system of $\mathbf{V}_{\infty, \sigma}$, together with its weight filtration is supposed to come from a local system over $\mathbb{Z}$. This hypothesis 
is automatically satisfied if $\mathbf{V}_{\infty, \sigma}$ is unipotent. It implies that the local system, tensored with $\mathbb{Q}_{l}$, can be interpreted as a lisse $l$-adic sheaf on $X_{\sigma}$ (compare [FK], I, §11).

d) $I_{l, \bar{\sigma}}$ is an isomorphism

$$
F_{\mathcal{O}}\left(\mathbf{V}_{\infty,\left.\bar{\sigma}\right|_{k}}\right) \otimes_{\mathbb{Q}} \mathbb{Q}_{l} \longrightarrow \bar{\sigma}^{*} F_{l}\left(\mathbf{V}_{l}\right)
$$

of weight-filtered $l$-adic sheaves on $X_{\sigma}$. Here, $F_{\mathcal{O}}$ and $F_{l}$ are suitably defined forgetful functors.

e) $I_{D R, \sigma}$ is a horizontal isomorphism

$$
F_{\mathcal{O}}^{\prime}\left(\mathbf{V}_{\infty, \sigma}\right) \longrightarrow \mathbf{V}_{D R} \otimes_{k, \sigma} \mathbb{C}
$$

of bifiltered vector bundles on $X_{\sigma}(\mathbb{C})$. Again, $F_{\mathcal{O}}^{\prime}$ is a suitable forgetful functor.

It follows that the filtrations in b) are finite and that the Hodge filtration in b) satisfies Griffiths-transversality:

$$
\nabla \mathcal{F}^{p} \subset \mathcal{F}^{p-1} \otimes_{\mathcal{O}_{X}} \Omega_{X / k}^{1} \quad \text { for all } p \in \mathbb{Z} .
$$

f) Let $c: \mathbb{C} \rightarrow \mathbb{C}$ denote complex conjugation.

For any $\sigma: k \hookrightarrow \mathbb{C}$, conjugation defines a diffeomorphism

$$
c_{\sigma}: X_{\sigma}(\mathbb{C}) \longrightarrow X_{c \circ \sigma}(\mathbb{C}) \text {. }
$$

For a variation of $\mathbb{Q}-M H S \mathbf{W}$ on $X_{c \circ \sigma}(\mathbb{C})$, we define a variation $c_{\sigma}^{*}(\mathbf{W})$ on $X_{\sigma}(\mathbb{C})$ as follows: the local system and the weight filtration are the pull backs via $c_{\sigma}$ of the local system and the weight filtration on $\mathbf{W}$, and the Hodge filtration is the pull back of the conjugate of the Hodge filtration on $\mathbf{W}$.

$c_{\sigma}^{*}$ preserves admissibility.

$I_{\infty, \sigma}$ is an isomorphism of variations of $\mathbb{Q}-M H S$

$$
\mathbf{V}_{\infty, \sigma} \longrightarrow c_{\sigma}^{*}\left(\mathbf{V}_{\infty, c \circ \sigma}\right)
$$

such that $c_{c \circ \sigma}^{*}\left(I_{\infty, \sigma}\right)=I_{\infty, c \circ \sigma}^{-1}$.

For $\rho \in G_{k}$ we suppose that $I_{l, \bar{\sigma} \rho}=\bar{\sigma}^{*}\left(\operatorname{can}_{\rho}\right) \circ I_{l, \bar{\sigma}}$. Here, $\operatorname{can}_{\rho}$ denotes the isomorphism $F_{l}\left(\mathbf{V}_{l}\right) \longrightarrow \rho^{*} F_{l}\left(\mathbf{V}_{l}\right)$ given by the fact that $F_{l}\left(\mathbf{V}_{l}\right)$ comes 
from $X$.

Furthermore, we require the following:

For each $\sigma$, let $c_{\infty, \sigma}$ be the antilinear involution of $F_{\text {diff. }}\left(\mathbf{V}_{\infty, \sigma}\right)$, the $C^{\infty}$-bundle underlying $\mathbf{V}_{\infty, \sigma}$, given by complex conjugation of coefficients. Likewise, let $c_{D R, \sigma}$ be the antilinear isomorphism

$$
F_{\text {diff. }}\left(\mathbf{V}_{\infty, \sigma}\right) \longrightarrow c_{\sigma}^{-1}\left(F_{\text {diff. }}\left(\mathbf{V}_{\infty, c \circ \sigma}\right)\right)
$$

given by complex conjugation of coefficients on the right hand side of the isomorphism in e). Our requirement is the validity of the formula

$$
F_{\text {diff. }}\left(I_{\infty, \sigma}\right)=c_{D R, \sigma}{ }^{\circ} c_{\infty, \sigma}=c_{\sigma}^{-1}\left(c_{\infty, c \circ \sigma}\right)^{\circ} c_{D R, \sigma}
$$

Example: In order to make conditions d)-f) transparent, we consider the mixed system $\mathbb{Q}(1)$ on $\operatorname{Spec}(k)$ :

The data a)-c) are given by the usual constructions.

For d), note that the underlying integral structures are given by the "local system on $\operatorname{Spec}(\mathbb{C}) " 2 \pi i \cdot \mathbb{Z}$ on the left hand side and the projective system $\mathbb{Z}_{l}(1)$ of $l^{n}$-th roots of unity in $\overline{\mathbb{Q}}$. The embedding $\bar{\sigma}: \overline{\mathbb{Q}} \hookrightarrow \mathbb{C}$ maps a topological generator $\gamma$ of $\mathbb{Z}_{l}(1)$ to the projective system $\left(\left(e^{\frac{2 \pi i}{l^{n}}}\right)_{n \in \mathbb{Z}}\right)^{m}$ for some number $m \in \mathbb{Z}_{l}^{*}$, and the isomorphism

$$
\left(2 \pi i \cdot \mathbb{Z} / l^{n} \mathbb{Z}\right)_{n \in \mathbb{Z}} \stackrel{\sim}{\longrightarrow}\left(\mu_{l^{n}}\right)_{n \in \mathbb{Z}}
$$

is given by sending $z$ to $\gamma^{\frac{z}{m}}$.

The isomorphisms $I_{\infty, \sigma}$ are given by the identity.

For $n \in \mathbb{Z}$, we let $\mathbb{Q}(n):=\mathbb{Q}(1)^{\otimes n}$. Also, for a scheme $X / k$ as above, we denote by $\mathbb{Q}(n)$ the pull-back via the structural morphism of the mixed system $\mathbb{Q}(n)$ on $\operatorname{Spec}(k)$.

Definition (continued): The last condition we impose is the existence of a system of polarizations: there are morphisms

$$
\begin{aligned}
\operatorname{Gr}_{n}^{W} \mathbf{V}_{l} \otimes_{\mathbb{Q}_{l}} \operatorname{Gr}_{n}^{W} \mathbf{V}_{l} & \rightarrow \mathbb{Q}_{l}(-n), l \in \mathbb{N} \text { prime }, n \in \mathbb{Z}, \\
\operatorname{Gr}_{n}^{W} \mathbf{V}_{D R} \otimes_{\mathcal{O}_{X}} \operatorname{Gr}_{n}^{W} \mathbf{V}_{D R} & \rightarrow \mathbb{Q}_{D R}(-n), n \in \mathbb{Z}
\end{aligned}
$$

of $l$-adic sheaves and flat vector bundles on $X$, and polarizations

$$
\operatorname{Gr}_{n}^{W} \mathbf{V}_{\infty, \sigma} \otimes_{\mathbb{Q}} \operatorname{Gr}_{n}^{W} \mathbf{V}_{\infty, \sigma} \rightarrow \mathbb{Q}(-n), \sigma: k \hookrightarrow \mathbb{C}, n \in \mathbb{Z}
$$


of variations of $\mathbb{Q}-M H S$ such that the $I_{l, \bar{\sigma}}, I_{D R, \sigma}$ and $I_{\infty, \sigma}$ and the corresponding morphisms of the mixed system $\mathbb{Q}(-n)$ form commutative diagrams.

ii) $U M S_{\mathbb{Q}}^{s}(X)$ is the full subcategory of objects admitting a filtration, whose graded objects come from $\operatorname{Spec}(k)$.

If $X$ is geometrically connected, then $M S_{\mathbb{Q}}^{s}(X)$ and $U M S_{\mathbb{Q}}^{s}(X)$ are Tannakian categories. As we shall see, results analogous to 2.6, 2.7 and 2.9 hold.

Remarks: a) Observe that a polarization on a pure Hodge structure induces a polarization on any subquotient. In particular, any subquotient of a pure polarizable Hodge structure enjoys the property of "self-duality up to twist".

We don't expect a concept similar to graded-polarizability to exist for Galois modules.

However, the definition of graded-polarizability, that we introduced for mixed systems, is well behaved. As in the Hodge-theoretic context it ensures that there are no non-trivial extensions of pure objects of the same weight.

b) As suggested by the notation, the category $M S_{\mathbb{Q}}^{s}(X)$ does not depend on the base field $k$ : if we consider the Grothendieck restriction

$$
X \longrightarrow \operatorname{Spec}(k) \longrightarrow \operatorname{Spec}(\mathbb{Q}),
$$

we get a smooth, separated scheme $Z$ of finite type over $\mathbb{Q}$ and a canonical isomorphism between $M S_{\mathbb{Q}}^{s}(X)$ and $M S_{\mathbb{Q}}^{s}(Z)$.

c) For an extension $K / k$ of number fields, we get a natural faithful forgetful functor

$$
M S_{\mathbb{Q}}^{s}(X) \longrightarrow M S_{\mathbb{Q}}^{s}\left(X_{K}\right) .
$$

It identifies $M S_{\mathbb{Q}}^{s}(X)$ with the category of descent data in $M S_{\mathbb{Q}}^{s}\left(X_{K}\right)$.

In order to be able to define the de Rham-version of $\mathcal{G} e n_{x}$, we prove the following result:

Lemma 2.10: Let $L / K$ be an extension of fields of characteristic zero, $S / K$ a locally noetherian scheme, $\mathcal{F}$ a coherent sheaf on $S$ and $\mathcal{G}$ a coherent subsheaf of $\mathcal{F}_{L}:=\mathcal{F} \otimes_{K} L$, that is invariant under all automorphisms of $L$ over $K$. Then $\mathcal{G}$ descends to a coherent subsheaf of $\mathcal{F}$. 
Proof: Because of the coherence of $\mathcal{G}$, and by usual Galois descent and induction, we may assume that $L=K(X)$. In order to get a descent datum for $\mathcal{G}$, we need to show that under the identification of $p_{1}^{*} \mathcal{F}_{K(X)}$ and $p_{2}^{*} \mathcal{F}_{K(Y)}$, where $p_{1}$ and $p_{2}$ are the morphisms

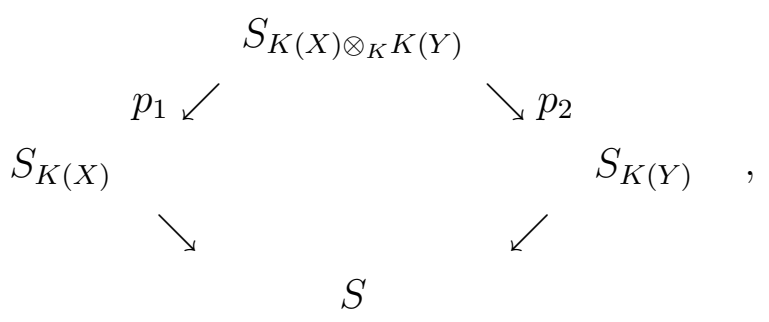

the sheaves $p_{1}^{*} \mathcal{G}$ and $p_{2}^{*} \mathcal{G}$ correspond.

Observe that $K(X) \otimes_{K} K(Y)$ is the ring obtained by localizing $K[X, Y]$ at the multiplicative subset $\{f \cdot g \mid f \in K[X], g \in K[Y]\}$. It is integral, noetherian of dimension one, and its fraction field is $K(X, Y)$.

Let $R:=K(X) \otimes_{K} K(Y)^{\mathrm{alg}}$, where $K(Y)^{\mathrm{alg}}$ is the algebraic closure of $K(Y)$. Let $Z$ denote the reduced closed subscheme of the points $s$ of $S_{R}$ where we have $\left(p_{1}^{*} \mathcal{G}\right)_{s} \neq\left(p_{2}^{*} \mathcal{G}\right)_{s}$.

Now observe that the maximal ideals of $R$ are in bijective correspondence with the non-constant functions in $K(Y)^{\text {alg }}$. It follows from our hypothesis that $Z$ must be contained in the generic fibre of $S_{R} \rightarrow \operatorname{Spec}(R)$, and hence that $Z$ is empty.

q.e.d.

Recall ([D1], II, Théorème 5.9) that any local system over $\mathbb{C}$ on the set $U(\mathbb{C})$ of $\mathbb{C}$-valued points of a smooth complex variety $U$, i.e., any vector bundle with a flat connection, is canonically equipped with an algebraic structure. The algebraic connection is regular at infinity. The following result is essentially a consequence of Schmid's Nilpotent Orbit Theorem ([Sch], Theorem 4.9):

Theorem 2.11: ([Ka], Proposition 1.11.3.)

Let $U$ be a smooth complex variety, $\mathbf{V}$ an admissible variation of Hodge structure on $U$.

Then the Hodge filtration is a filtration by subbundles, that are algebraic with respect to the canonical algebraic structure on the local system underlying $\mathbf{V}$.

We are now in a position to define the de Rham-version of $\mathcal{G} e n_{x}$ : fix an embedding $\sigma$ of $k$ into $\mathbb{C}$. By [D1], II, Théorème 5.9 and the previous theorem, both the weight and the Hodge filtrations of $\mathcal{G} e n_{x, \infty, \sigma}$ are algebraic. In [D4], 
10.36-10.43, it is proven that the pro-vector bundle underlying $\mathcal{G} e n_{x, \infty, \sigma}$ and its flat connection carry a canonical $k$-structure. It is given by the fact that the base change by $\mathbb{C}$ of the Tannaka dual of the category of unipotent vector bundles with integrable connection on $X$ coincides with the Tannaka dual of the analogous category of bundles on $X_{\sigma}$, i.e., the Tannaka dual of the category of unipotent representations of $\pi_{1}\left(X_{\sigma}(\mathbb{C}), \bar{x}\right)$, which by 2.2.b) coincides with $W\left(\pi_{1}\left(X_{\sigma}(\mathbb{C}), \bar{x}\right)\right) \hat{\otimes}_{\mathbb{Q}} \mathbb{C}$. By the same process as in the proof of 2.8 , it is possible to describe the $k$-structure of the weight filtration.

So it makes sense to speak of $\mathcal{G} e n_{x, \infty, \sigma}^{\tau}$, the variation on $X_{\sigma}(\mathbb{C})$ conjugate to $\mathcal{G} e n_{x, \infty, \sigma}$ under $\tau \in \operatorname{Aut}(\mathbb{C} / k)$. Its underlying local system and weight filtration coincide with those of $\mathcal{G} e n_{x, \infty, \sigma}$. Clearly $\mathcal{G} e n_{x, \infty, \sigma}^{\tau}$ is pro-unipotent and admissible. In order to show that the Hodge filtration coincides with that of $\mathcal{G e n} n_{x, \infty, \sigma}$, observe that by 2.6 the element 1 of the fibre $\hat{\mathfrak{U}}_{\bar{x}}$ of $\mathcal{G} e n_{x, \infty, \sigma}$ at $\bar{x}$ induces a natural isomorphism of functors on $U \operatorname{Var}_{\mathbb{Q}}\left(X_{\sigma}\right)$

$$
\operatorname{Hom}\left(\mathcal{G e n}_{x, \infty, \sigma}, \quad\right) \stackrel{\sim}{\longrightarrow}\left(\mathbf{V} \mapsto\left(W_{0, \mathbb{Q}} \cap F^{0}\right) \mathbf{V}_{\bar{x}}\right) .
$$

If we apply this observation to the element 1 of the fibre of $\mathcal{G e n} n_{x, \infty, \sigma}^{\tau}$ at $\bar{x}$, we see that the isomorphism $1 \mapsto 1$ of the underlying local systems of $\mathcal{G} e n_{x, \infty, \sigma}$ and $\mathcal{G} e n_{x, \infty, \sigma}^{\tau}$ respects the Hodge filtrations as well. By 2.10, the Hodge filtration of $\mathcal{G} e n_{x, \infty, \sigma}$ descends to $k$.

Arguments similar to the ones used above show that the object $\mathcal{G} e n_{x, D R}$ thus defined is independent of the choice of $\sigma$.

Remarks: a) The fact that the weight and Hodge filtrations of $\mathcal{G} e n_{x, \infty, \sigma}$ descend to the base field was already observed by Wojtkowiak: see [Wo1], Theorem E. b) In fact, Wojtkowiak shows ([Wo1], [Wo2]) that the Hodge, $l$-adic and de Rham versions of $\mathcal{G} e n_{x}$ appear as relative cohomology objects for a morphism of certain smooth, simplicial schemes. So if we follow the definition of the category of sheaves "of geometric origin" proposed in the introduction of [Wo1], the generic sheaves $\mathcal{G} e n_{x}$ in fact belong to that category.

It remains to show that the various versions of $\mathcal{G} e n_{x}$ fit together to form a pro-mixed system of smooth sheaves on $X$. The compatibility of the weight filtrations follows from an observation similar to the above: they are compatible with the multiplicative structures of the fibres at $x$, and they correspond on the cohomology groups (see $[\mathrm{J}], \S 3$ ). 
Similar arguments show that the morphisms induced by complex conjugation give rise to isomorphisms $I_{\infty, \sigma}$, which behave as required. So up to the existence of a system of polarizations, we have checked all of the axioms.

Theorem 2.12: Let $X$ be a smooth, separated, geometrically connected scheme of finite type over a number field $k$, and let $x \in X(k)$.

a) $\mathcal{G} e n_{x}:=\left(\mathcal{G e n}_{x, l}, \mathcal{G} e n_{x, D R}, \mathcal{G} e n_{x, \infty, \sigma}, I_{l, \bar{\sigma}}, I_{D R, \sigma}, I_{\infty, \sigma} \mid l, \sigma, \bar{\sigma}\right)$ is a pro-object of $U M S_{\mathbb{Q}}^{s}(X)$.

b) Let $P_{\bar{x}}$ be the Tannaka dual of $U M S_{\mathbb{Q}}^{s}(X)$ with respect to

"fibre at $\bar{x} " \circ\left(\right.$ projection to the $\left(\infty, \sigma_{0}\right)$-component):

$$
U M S_{\mathbb{Q}}^{s}(X) \longrightarrow U \operatorname{Var}_{\mathbb{Q}}\left(X_{\sigma_{0}}\right) \longrightarrow \operatorname{Vec}_{\mathbb{Q}}
$$

for some choice of $\sigma_{0}: k \hookrightarrow \mathbb{C}$,

$$
P_{\bar{x}} \underset{x}{\stackrel{\pi}{\rightleftarrows}} G
$$

the morphisms induced by $\pi^{*}$ and "fibre at $x$ ", where $G$ is the Tannaka dual of $M S_{\mathbb{Q}}^{s}(\operatorname{Spec}(k))$ with respect to

$$
\text { "forget" ○(projection to the }\left(\infty, \sigma_{0}\right) \text {-component). }
$$

Then the natural morphism

$$
W\left(\pi_{1}\left(X_{\sigma_{0}}(\mathbb{C}), \bar{x}\right)\right) \stackrel{\eta_{\bar{x}}}{\longrightarrow} W_{\bar{x}}:=\operatorname{ker}(\pi)
$$

is an isomorphism.

c) The functor

$$
\begin{aligned}
& U M S_{\mathbb{Q}}^{s}(X) \longrightarrow\left\{\begin{array}{l}
\text { objects } \mathbf{H} \text { of } M S_{\mathbb{Q}}^{s}(\operatorname{Spec}(k)) \\
\text { together with a morphism } \\
\text { of mixed systems on } \operatorname{Spec}(k) \\
\hat{\mathfrak{U}}_{\bar{x}} \longrightarrow \text { End }(\mathbf{H}) \text { respecting } \\
\text { the algebra structure }
\end{array}\right\}, \\
& \mathbf{V} \longmapsto \mathbf{V}_{\bar{x}}
\end{aligned}
$$

is an equivalence of categories.

Here, as usual, $\hat{\mathfrak{U}}_{\bar{x}}$ carries the mixed structure of the fibre at $x$ of $\mathcal{G} e n_{x}$. 
Proof: We prove a), leaving b) and c) to the reader.

It suffices to show graded-polarizability of the restriction of our system to a dense open subset $U$ of $X$ : any polarization will automatically extend to the whole of $X$ because the respective fundamental groups of $U$ surject onto those of $X$ and because of 2.2.a), 2.6, 2.9.c) and [D4], Corollaire 10.43. The remark following the definition of $U M S_{\mathbb{Q}}^{s}(X)$ allows us to assume that $X$ is affine.

Then we have a mixed system on $\operatorname{Spec}(k)$

$$
H^{1}(\bar{X}, \mathbb{Q}(0)):=\left(H^{1}\left(\bar{X}, \mathbb{Q}_{l}(0)\right), H_{D R}^{1}(X), H^{1}\left(X_{\sigma}(\mathbb{C}), \mathbb{Q}(0)\right) \mid l, \sigma\right),
$$

where the polarizations on the graded parts are constructed as follows: choose a smooth projective compactification $Z / k$ of $X$ such that $Z \backslash X$ is a divisor with normal crossings. The Leray spectral sequences for $\bar{X} \hookrightarrow \bar{Z}$, for $X \hookrightarrow Z$ and the $X_{\sigma} \hookrightarrow Z_{\sigma}$ give an interpretation of the graded parts of the above system as subquotients of cohomology systems of smooth projective varieties. For any such, choose a hyperplane section. It defines an algebraic correspondence, which in particular defines a compatible decomposition of the cohomology system into primitive components. On these, polarizations are given by the composition of the successive intersection with the class of the hyperplane section and cup product. For details, see [D2], 3.2 and 2.2.6.

The weight-graded objects of $\mathcal{G} e n_{x}$ are subquotients of direct sums of tensor powers of $\pi^{*} H^{1}(\bar{X}, \mathbb{Q}(0))^{\vee}$ : the map

$$
\pi_{2 l+m=n}^{*}\left(\mathrm{Gr}_{-1}^{w} H^{1}(\bar{X}, \mathbb{Q}(0))^{\vee}\right)^{\otimes m} \otimes_{\mathbb{Q}}\left(W_{-2} H^{1}(\bar{X}, \mathbb{Q}(0))^{\vee}\right)^{\otimes l} \rightarrow \mathrm{Gr}_{-n}^{w} \mathcal{G} e n_{x}
$$

is well-defined and surjective.

So again by the remark following the definition of $U M S_{\mathbb{Q}}^{s}(X)$, the system $\mathcal{G} e n_{x}$ is graded-polarizable.

q.e.d. 


\section{$\S 3$ The generic pro-sheaf: the relative case}

In this paragraph, we aim for statements analogous to 2.7, 2.9.b) and 2.12.b) in the relative case, i.e., the case of a suitably regular morphism

$$
\pi: X \rightarrow Y
$$

While the results of [SGA1], Exp. XIII are strong enough to let the proofs of 2.8 and 2.9 carry over almost verbatim, the situation in the Hodge-theoretic setting requires a bit more work.

The main step, as suggested by the proof of 2.7, is to show that the relative version of $\hat{\mathfrak{U}}_{\bar{x}}$ underlies an admissible pro-variation of $M H S$ on $X$. We conclude the paragraph by a characterization of the relative version of $\mathcal{G} e n_{x}$ by a universal property $(3.5,3.6 . d))$, which we regard as the central result of this work.

Again, let $l$ be a prime number, either $F / \mathbb{Q}_{l}$ finite or $F=\overline{\mathbb{Q}_{l}}, k$ a number field, $\pi: X \rightarrow Y$ a morphism of type $(S)$ of schemes over $k$, which we define to be a smooth morphism with geometrically connected fibres between smooth, separated, geometrically connected schemes of finite type over $k, \pi$ being compactifiable in such a way that $X$ is the complement of a relative divisor with normal crossings in a smooth, projective $Y$-scheme. Following [BL], 1.1.1, we define:

Definition: $\pi-U \mathrm{Et}_{F}^{l, m}(X)$ is the full subcategory of those $\mathbf{V} \in \mathrm{Et}_{F}^{l, m}(X)$ admitting a filtration, whose graded objects lie in $\pi^{*}\left(\mathrm{Et}_{F}^{l, m}(Y)\right)$.

Again, since $\pi$ is smooth, if such a filtration exists, the weight filtration will have the same property, as follows from [D3], Théorème 6.1.2, generic base change ([SGA4 1/2], Th. finitude, Théorème 1.9) and [D3], Corollaire 3.3.5.

Objects in $\pi-U \mathrm{Et}_{F}^{l, m}(X)$ will be called $\pi$-unipotent, or relatively unipotent, mixed lisse $F$-sheaves.

Until Theorem 3.1, we shall also assume that $\pi$ admits a section $i: Y \hookrightarrow X$. Afterwards, this assumption will be weakened slightly. Fix $y \in Y(\bar{k})$ and let $x:=i(y)$.

Because $\pi$ is of type $(S)$, we may apply [SGA1], Exp. XIII, Proposition 4.3 and Exemples 4.4 to conclude that there is a split exact sequence

$$
1 \longrightarrow \pi_{1}\left(\bar{X}_{\bar{y}}, \bar{x}\right) \longrightarrow \pi_{1}(X, \bar{x}) \stackrel{\pi}{\stackrel{i}{\rightleftarrows}} \pi_{1}(Y, \bar{y}) \longrightarrow 1
$$


So if a mixed lisse $F$-sheaf on $X$ is relatively unipotent then the induced representation of $\pi_{1}\left(\bar{X}_{\bar{y}}, \bar{x}\right)$ is unipotent. It will be shown in the proof of 3.2 that the converse also holds. Again, $\pi-U \operatorname{Et}_{F}^{l, m}(X)$ is a neutral Tannakian category over $F$.

The splitting $i$ defines a continuous action of $\pi_{1}(Y, \bar{y})$ on

$$
\hat{\mathfrak{U}}\left(\mathfrak{w}_{l}\left(\pi_{1}\left(\bar{X}_{\bar{y}}, \bar{x}\right)\right)\right) \hat{\otimes}_{\mathbb{Q}_{l}} F=: \hat{\mathfrak{U}}_{\pi, \bar{x}} .
$$

So $\hat{\mathfrak{U}}_{\pi, \bar{x}}$ carries the structure of a lisse pro- $F$-sheaf on $Y$. As before, we define a continuous representation of

$$
\pi_{1}(X, \bar{x})=\pi_{1}\left(\bar{X}_{\bar{y}}, \bar{x}\right) \rtimes \pi_{1}(Y, \bar{y})
$$

as follows:

the underlying vector space is $\hat{\mathfrak{U}}_{\pi, \bar{x}}$, the fundamental group $\pi_{1}\left(\bar{X}_{\bar{y}}, \bar{x}\right)$ acts by multiplication, and $\pi_{1}(Y, \bar{y})$ acts as above.

This defines a lisse pro- $F$-sheaf $\mathcal{G} e n_{i}$ on $X$. By construction, the induced representation of $\pi_{1}\left(\bar{X}_{\bar{y}}, \bar{x}\right)$ is pro-unipotent.

Theorem 3.1: $\mathcal{G e n}_{i}$ is an object of pro- $\pi-U \operatorname{Et}_{F}^{l, m}(X)$.

Proof: The assumptions on $\pi$ are sufficient to construct extensions

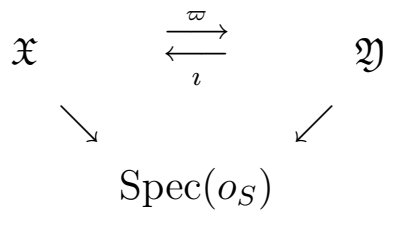

of $\pi$ and $i$ for a suitably chosen finite set $S \subset \operatorname{Spec}\left(o_{k}\right)$ containing the primes dividing $l$, such that [SGA1], Exp. XIII, Proposition 4.3 and Exemples 4.4 are applicable.

So as in the proof of 2.8 , the representation of $\pi_{1}(X, \bar{x})$ factors through

$$
\pi_{1}^{\prime}(\mathfrak{X}, \bar{x}):=\pi_{1}(\mathfrak{X}, \bar{x}) / N
$$

where $\operatorname{ker}\left(\varpi: \pi_{1}(\mathfrak{X}, \bar{x}) \rightarrow \pi_{1}(\mathfrak{Y}, \bar{y})\right) / N$ is the largest pro- $l$ quotient of $\operatorname{ker}(\varpi)$. The weight filtration on $\hat{\mathfrak{U}}_{\pi, \bar{x}}$ as defined in the proof of 2.8 is stable under the action of $\pi_{1}(X, \bar{x})$, and the graded quotients are pure subquotients of direct sums of the $H^{1}\left(\bar{X}_{\bar{y}}, F(0)\right)^{\otimes(-n)}=\left(R^{1} \varpi_{*} F_{\mathfrak{X}}(0)\right)_{\bar{y}}^{\otimes(-n)}$.

The representation of $\pi_{1}(X, \bar{x})$ on these quotients corresponds precisely to the 
representation of $\pi_{1}(\mathfrak{X}, \bar{x})$ on $\varpi^{*}\left(R^{1} \varpi_{*} F_{\mathfrak{X}}(0)\right)^{\otimes(-n)}$, so the corresponding sheaves are mixed. As their stalks at $\bar{x}$ are pure, they are pure altogether. q.e.d. For the independence of $\mathcal{G} e n_{i}$ of the choice of $y$, see Theorem 3.5.iii).

Corollary 3.2: Assume that after a finite étale covering $Y^{\prime} \rightarrow Y, \pi$ admits a section

$$
i: Y^{\prime} \rightarrow X^{\prime}:=X \times_{Y} Y^{\prime} .
$$

Fix $y^{\prime} \in Y^{\prime}(\bar{k})$, and let $x^{\prime}:=i\left(y^{\prime}\right), x:=p r_{1}\left(x^{\prime}\right)$ and $y:=\pi(x)$.

Let $P_{\bar{x}}$ be the Tannaka dual of $\pi-U \mathrm{Et}_{F}^{l, m}(X)$ with respect to

$$
\text { "fibre at } \bar{x} ": \pi-U \operatorname{Et}_{F}^{l, m}(X) \longrightarrow \operatorname{Vec}_{F},
$$

$G_{\bar{y}}$ the Tannaka dual of $\operatorname{Et}_{F}^{l, m}(Y)$ with respect to

$$
\text { "fibre at } \bar{y} ": \operatorname{Et}_{F}^{l, m}(Y) \longrightarrow \operatorname{Vec}_{F}
$$

$\pi: P_{\bar{x}} \rightarrow G_{\bar{y}}$ the morphism induced by $\pi^{*}$.

i) The natural morphism

$$
W_{l}\left(\pi_{1}\left(\bar{X}_{\bar{y}}, \bar{x}\right)\right) \hat{\otimes}_{\mathbb{Q}_{l}} F \stackrel{\eta_{\bar{x}}}{\longrightarrow} W_{\bar{x}}:=\operatorname{ker}(\pi)
$$

is an isomorphism.

ii) If $Y^{\prime}=Y$, then $i$ defines a section of $\pi: P_{\bar{x}} \rightarrow G_{\bar{y}}$, and the functor

$$
\begin{aligned}
& \pi-U \mathrm{Et}_{F}^{l, m}(X) \longrightarrow\left\{\begin{array}{l}
\text { objects of } \mathrm{Et}_{F}^{l, m}(Y) \\
\text { together with a } \\
\pi_{1}(Y, \bar{y}) \text {-equivariant } \\
\text { action of } i^{*} \mathcal{G} e n_{i}
\end{array}\right\}: \\
& \mathbf{V} \longmapsto i^{*} \mathbf{V}
\end{aligned}
$$

is an equivalence of categories.

Proof: The sequence

$$
W_{l}\left(\pi_{1}\left(\bar{X}_{\bar{y}}, \bar{x}\right)\right) \hat{\otimes}_{\mathbb{Q}_{l}} F \stackrel{\eta_{\bar{x}}}{\longrightarrow} P_{\bar{x}} \stackrel{\pi}{\longrightarrow} G_{\bar{y}} \longrightarrow 1
$$

is exact: $\pi$ is an epimorphism by 1.2.a), and $\operatorname{im}\left(\eta_{\bar{x}}\right)=W_{\bar{x}}$ by 1.4.a): in order to see that a representation $\mathbf{V}$ of $\pi_{1}(Y, \bar{y})$ factors over some $\pi_{1}(\mathfrak{Y}, \bar{y})$ if $\pi^{*} \mathbf{V}$ factors 
over some $\pi_{1}(\mathfrak{X}, \bar{x})$, we choose a fixed extension $\varpi$ of $\pi$ as in the proof of 3.1 and apply [SGA1], Exp. XIII, Proposition 4.3 and Exemples 4.4, together with [EGAIV,3], Théorème 8.8.2.

So if $Y^{\prime}=Y$, the statements follow from 3.1, 1.4.b) and 1.5. In the general case, we assume $Y^{\prime}$ to be a geometrically connected Galois covering of $Y$. If $P_{\bar{x}^{\prime}}$, $G_{\bar{y}^{\prime}}$ and $W_{\bar{x}^{\prime}}$ denote the corresponding objects on the level of $X^{\prime}$, we have an isomorphism

$$
W_{l}\left(\pi_{1}\left(\bar{X}_{\bar{y}}, \bar{x}\right)\right) \hat{\otimes}_{\mathbb{Q}_{l}} F \stackrel{\sim}{\longrightarrow} W_{\bar{x}^{\prime}} .
$$

It remains to show that $P_{\bar{x}^{\prime}} \rightarrow P_{\bar{x}}$ is a monomorphism: given a representation of $\pi_{1}\left(X^{\prime}, \bar{x}^{\prime}\right)$, we convince ourselves that the induced representation of $\pi_{1}(X, \bar{x})$ inherits relative unipotency, mixedness and graded-polarizability. So we may apply $1.2 . \mathrm{b})$.

q.e.d.

We now describe the Hodge-theoretic situation.

Let $F \subset \mathbb{R}$ be a field, $\pi: X \rightarrow Y$ a morphism of type $(S)$ of schemes over $\mathbb{C}$. $\bar{\pi}: \bar{X} \rightarrow \bar{Y}$ will denote the map on topological spaces underlying $\pi_{a n}$.

\section{Definition:}

i) $\operatorname{Var}_{F}(X)$ is the full subcategory of those objects of the category of gradedpolarizable variations of $F-M H S$ on $X$, that are admissible in the sense of $[\mathrm{Ka}]$.

ii) $\pi-U \operatorname{Var}_{F}(X)$ is the full subcategory of those $\mathbf{V} \in \operatorname{Var}_{F}(X)$ admitting a filtration, whose graded objects lie in $\pi^{*}\left(\operatorname{Var}_{F}(Y)\right)$.

From $[\mathrm{Ka}], \S 0$, we recall that admissibility of a graded-polarizable variation can be checked via the curve test.

It follows from [SZ], Corollary A.10 that the above categories are neutral Tannakian.

Now assume that $\pi$ admits a section $i: Y \hookrightarrow X$. Fix $y \in Y(\mathbb{C})$ and let $x:=i(y)$. Because $\pi$ is of type $(S)$, the continuous map $\bar{\pi}: \bar{X} \rightarrow \bar{Y}$ is locally trivial (compare [D1], II, 6.17). In particular, it is a weak fibration ([Sp], II, $\S 7$, Corollary 
14 and VII, $\S 2$, Definition after Corollary 4), so we have a split exact sequence

$$
1 \longrightarrow \pi_{1}\left(\bar{X}_{\bar{y}}, \bar{x}\right) \longrightarrow \pi_{1}(\bar{X}, \bar{x}) \stackrel{\pi}{\stackrel{\rightleftarrows}{\rightleftarrows}} \pi_{1}(\bar{Y}, \bar{y}) \longrightarrow 1
$$

We define a representation of

$$
\pi_{1}(\bar{X}, \bar{x})=\pi_{1}\left(\bar{X}_{\bar{y}}, \bar{x}\right) \rtimes \pi_{1}(\bar{Y}, \bar{y})
$$

as follows:

the underlying vector space is

$$
\hat{\mathfrak{U}}_{\pi, \bar{x}}:=\hat{\mathfrak{U}}\left(\mathfrak{w}\left(\pi_{1}\left(\bar{X}_{\bar{y}}, \bar{x}\right)\right)\right) \hat{\otimes}_{\mathbb{Q}} F
$$

$\pi_{1}\left(\bar{X}_{\bar{y}}, \bar{x}\right)$ acts by multiplication, and $\pi_{1}(\bar{Y}, \bar{y})$ acts by conjugation. This defines a pro-local system of $F$-vector spaces on $\bar{X}$. By construction, the induced representation of $\pi_{1}\left(\bar{X}_{\bar{y}}, \bar{x}\right)$ is pro-unipotent.

Given $y^{\prime} \in Y(\mathbb{C})$, there is a canonical isomorphism

$$
\left.\left(\hat{\mathfrak{U}}_{\pi, \bar{x}}\right)\right|_{\bar{X}_{\bar{y}^{\prime}}} \stackrel{\sim}{\longrightarrow} \operatorname{For}\left(\mathcal{G} e n_{i\left(y^{\prime}\right)}\right)
$$

where the right hand side denotes the pro-local system underlying the generic pro-unipotent variation on $X_{y^{\prime}}$ with basepoint $i\left(y^{\prime}\right)$. This isomorphism allows us to define weight and Hodge filtrations on $\hat{\mathfrak{U}}_{\pi, \bar{x}}$, at least fibrewise.

Theorem 3.3: The above data define an object $\mathcal{G}_{e n_{i}}$ of $\operatorname{pro}^{-} \pi-U \operatorname{Var}_{F}(X)$.

Proof: Our data provide an example of the path space variations considered in [HZ1], $\S 4$. In their notation,

$$
g: Y \longrightarrow S
$$

is $p_{1}: X \times_{Y} X \rightarrow X$ in ours, and the two sections $\sigma_{0}$ and $\sigma_{1}$ are taken to be $x \mapsto(x, i \circ \pi(x))$ and $x \mapsto(x, x)$ respectively. Then our data are precisely the ones called

$$
\left\{H_{0}\left(P_{\sigma_{0}(s), \sigma_{1}(s)} Y_{s} ; \mathbb{C}\right)\right\}_{s \in S}
$$

in [HZ1], Proposition 4.20.ii), as can be seen from [HZ1], Definition 4.21.ii). By [HZ1], Proposition 4.20.ii), they define a graded-polarizable variation of Q- $M H S$.

We now check the conditions of [Ka], (1.8) and (1.9).

If the image of a morphism $f: \Delta^{*} \rightarrow X$ of the punctured unit disc into $X$ is contained in a single fibre, then $f^{*}\left(\mathcal{G e} n_{i}\right)$ is admissible because $\mathcal{G} e n_{i\left(\pi \circ f\left(\Delta^{*}\right)\right)}$ is 
admissible.

Else we may replace $Y$ by $\Delta^{*}$, assuming that there is another section $f$ of $\pi$. But this is exactly the situation studied in [HZ1], $\S 6$. There, conditions (1.8.3) and (1.8.4) of [Ka] are proven. Condition (1.8.2) of [Ka], i.e., quasiunipotency at infinity, which in [SZ], (3.13) was not yet formulated, follows from the corresponding statement for $R^{1} \bar{\pi}_{*}(\mathbb{C})$. But this is a consequence of Brieskorn's Monodromy Theorem ([D1], III, Théorème 2.3). $\quad$ q.e.d.

Corollary 3.4: Assume that after a finite étale covering $Y^{\prime} \rightarrow Y, \pi$ admits a section

$$
i: Y^{\prime} \longrightarrow X^{\prime}:=X \times_{Y} Y^{\prime} .
$$

Fix $y^{\prime} \in Y^{\prime}(\mathbb{C})$, and let $x^{\prime}:=i\left(y^{\prime}\right), x:=p r_{1}\left(x^{\prime}\right)$ and $y:=\pi(x)$.

Let $P_{\bar{x}}$ be the Tannaka dual of $\pi-U \operatorname{Var}_{F}(X)$ with respect to

$$
\text { "fibre at } \bar{x} ": \pi-U \operatorname{Var}_{F}(X) \longrightarrow \operatorname{Vec}_{F},
$$

$G_{\bar{y}}$ the Tannaka dual of $\operatorname{Var}_{F}(Y)$ with respect to

$$
\text { "fibre at } \bar{y} ": \operatorname{Var}_{F}(Y) \longrightarrow \operatorname{Vec}_{F} \text {, }
$$

$\pi: P_{\bar{x}} \rightarrow G_{\bar{y}}$ the morphism induced by $\pi^{*}$.

i) The natural morphism

$$
W\left(\pi_{1}\left(\bar{X}_{\bar{y}}, \bar{x}\right)\right) \hat{\otimes}_{\mathbb{Q}} F \stackrel{\eta_{\bar{x}}}{\longrightarrow} W_{\bar{x}}:=\operatorname{ker}(\pi)
$$

is an isomorphism.

ii) If $Y^{\prime}=Y$, then $i$ defines a section of $\pi: P_{\bar{x}} \rightarrow G_{\bar{y}}$, and the functor

$$
\begin{aligned}
& \pi-U \operatorname{Var}_{F}(X) \longrightarrow\left\{\begin{array}{l}
\text { admissible graded-polarizable variations } \\
\mathbf{W} \text { of } F-M H S \text { on } Y \text { together with } \\
\text { a morphism } i^{*} \mathcal{G e n}_{i} \rightarrow \underline{\operatorname{End}}_{F}(\mathbf{W}) \text { of pro-- } \\
\text { variations on } Y \text { respecting the algebra } \\
\text { structure }
\end{array}\right\}, \\
& \mathbf{V} \longmapsto i^{*} \mathbf{V}
\end{aligned}
$$

is an equivalence of categories.

Proof: left to the reader.

q.e.d. 
As in $\S 2$, it is only a formal matter to write down statements analogous to 3.1-3.4 for mixed systems. Assume that $\pi$ is of type $(S)$ and admits a section $i$ sending $y$ to $x$. We have to study the dependence of $\mathcal{G} e n_{i}$ on $y$ : as usual, the choice of a path in the Hodge-theoretic setting or a chain of specialization maps connecting $\bar{y}$ and $\bar{y}^{\prime}$ in the $\lambda$-adic setting gives an isomorphism

$$
\mathcal{G e n}_{i, \bar{y}^{\prime}} \stackrel{\sim}{\longrightarrow} \mathcal{G}_{i, \bar{y}}
$$

sending $1 \in \Gamma\left(Y, i^{*} \mathcal{G} e n_{i, \bar{y}^{\prime}}\right)$ to $1 \in \Gamma\left(Y, i^{*} \mathcal{G} e n_{i, \bar{y}}\right)$.

Somewhat surprisingly, this isomorphism is in fact independent of our choices, as follows from the next result, which holds in the Hodge theoretic as well as in the $l$-adic context:

\section{Theorem 3.5:}

i) The natural transformation of functors from $\pi-U \operatorname{Var}_{\mathbb{Q}}(X)$ to $\operatorname{Var}_{\mathbb{Q}}(Y)$ (resp. from $\pi-U \mathrm{Et}_{\mathbb{Q}_{l}}^{l, m}(X)$ to $\mathrm{Et}_{\mathbb{Q}_{l}}^{l, m}(Y)$ )

$$
\begin{aligned}
e v: \pi_{*} \underline{\operatorname{Hom}}\left(\mathcal{G e n}_{i},-\right) & \longrightarrow i^{*}, \\
\varphi & \longmapsto\left(i^{*} \varphi\right)(1)
\end{aligned}
$$

is an isomorphism.

Observe that the direct system

$$
c d\left(\pi_{*} \underline{\operatorname{Hom}}\left(\mathcal{G e n}_{i} / \mathfrak{a}^{n}, \mathbf{V}\right)\right)_{n \in \mathbb{N}},
$$

where $\mathfrak{a}$ denotes the augmentation ideal of $\mathcal{G} e n_{i}$, becomes constant for any $\mathbf{V} \in \pi-U \operatorname{Var}_{\mathbb{Q}}(X)$ (resp. $\left.\pi-U \mathrm{Et}_{\mathbb{Q}_{l}}^{l, m}(X)\right)$. This constant value is denoted by

$$
\pi_{*} \underline{\operatorname{Hom}}\left(\mathcal{G e n}_{i}, \mathbf{V}\right)
$$

ii) The natural transformation of functors from the category of relatively unipotent local systems (resp. $l$-adic lisse sheaves) on $\bar{X}$ to the category of local systems (resp. $l$-adic lisse sheaves) on $\bar{Y}$

$$
\begin{aligned}
\operatorname{For}_{\mathbb{Q}_{(l)}}(e v): \bar{\pi}_{*} \underline{\operatorname{Hom}}\left(\operatorname{For}_{\mathbb{Q}_{(l)}}\left(\mathcal{G e n}_{i}\right),{ }_{-}\right) & \longrightarrow \bar{i}^{*} \\
\varphi & \longmapsto\left(\bar{i}^{*} \varphi\right)(1)
\end{aligned}
$$

is an isomorphism.

iii) The pair $\left(\operatorname{For}_{\mathbb{Q}_{(l)}}(\mathcal{G e n}), 1\right)$ admits no non-trivial automorphisms. 
Proof: Let $P=W \rtimes G$ be a semidirect product of pro-algebraic groups over $\mathbb{Q}_{(l)}$. Assume that $W$ is a countable projective limit of unipotent group schemes, and let 1 be the unit element of the completed universal envelope $\hat{\mathfrak{U}}($ Lie $W)$. Then the natural transformation of functors from $\operatorname{Rep}_{\mathbb{Q}_{(l)}}(P)$ to $\operatorname{Rep}_{\mathbb{Q}_{(l)}}(G)$

$$
\begin{aligned}
e v: \operatorname{Hom}_{W}\left(\hat{\mathfrak{U}}(\operatorname{Lie} W),{ }_{-}\right) & \longrightarrow \operatorname{res}_{P}^{G}, \\
\varphi & \longmapsto\left(\operatorname{res}_{P}^{G}(\varphi)\right)(1)
\end{aligned}
$$

is an isomorphism.

q.e.d.

Remark: 3.5.i) still holds in the $l$-adic context when we consider the categories $\pi-U \mathrm{Et}_{\mathbb{Q}_{l}}^{l}(X)$ and $\mathrm{Et}_{\mathbb{Q}_{l}}^{l}(Y)$, i.e., remove the mixedness assumption.

Now, again assume $k$ is a number field and $\pi: X \rightarrow Y$ is of type $(S)$.

Definition: $\pi-U M S_{\mathbb{Q}}^{s}(X)$ is the full subcategory of $M S_{\mathbb{Q}}^{s}(X)$ of objects admitting a filtration, whose graded objects lie in $\pi^{*}\left(M S_{\mathbb{Q}}^{s}(Y)\right)$.

The de Rham version of $\mathcal{G e n}$ is constructed in a manner analogous to that of $\S 2$, Theorem 2.6 being replaced by 3.4.ii). In order to apply 2.10, we need to know in advance that the pro-vector bundle underlying $\mathcal{G} n_{i, \infty, \sigma}$ and its connection carry a canonical $k$-structure. This is provided by [D4], Corollaire 10.42.ii), applied to $F=\left\{R^{1} \pi_{*} \Omega_{X / Y}\right\}$, the vector bundle $R^{1} \pi_{*} \Omega_{X / Y}$ being equipped with the Gauß-Manin connection, which is flat and regular at infinity.

Remark: If we consider the categories $\operatorname{VB}(Y)$ and $\pi-U \operatorname{VB}(X)$ of flat vector bundles on $Y$ and $\pi$-unipotent flat vector bundles on $X$, whose connection is regular at infinity, then the flat vector bundle underlying $\mathcal{G} e n_{i, D R}$, which is a pro-object of $\pi-U \operatorname{VB}(X)$, together with the section 1 of $i^{*} \mathcal{G} e n_{i, D R}$, has a universal property similar to that of 3.5.i).

Theorem 3.6: Assume that after a finite étale covering $Y^{\prime} \rightarrow Y, \pi$ admits a section

$$
i: Y^{\prime} \longrightarrow X^{\prime}:=X \times_{Y} Y^{\prime} .
$$

Fix $y^{\prime} \in Y^{\prime}(\bar{k})$, and let $x^{\prime}:=i\left(y^{\prime}\right), x:=p r_{1}\left(x^{\prime}\right)$ and $y:=\pi(x)$.

a) If $Y^{\prime}=Y$, then

$$
\mathcal{G} e n_{i}:=\left(\mathcal{G e n}_{i, l}, \mathcal{G e n}_{i, D R}, \mathcal{G} e n_{i, \infty, \sigma}, I_{l, \bar{\sigma}}, I_{D R, \sigma}, I_{\infty, \sigma} \mid l, \sigma, \bar{\sigma}\right)
$$

is a pro-object of $\pi-U M S_{\mathbb{Q}}^{s}(X)$. 
b) Let $P_{\bar{x}}$ be the Tannaka dual of $\pi-U M S_{\mathbb{Q}}^{s}(X)$ with respect to "fibre at $\bar{x} " \circ\left(\right.$ projection to the $\left(\infty, \sigma_{0}\right)$-component) for some choice of $\sigma_{0}: k \hookrightarrow \mathbb{C}$, $G_{\bar{y}}$ the Tannaka dual of $M S_{\mathbb{Q}}^{s}(Y)$ with respect to "fibre at $\bar{y} " \circ\left(\right.$ projection to the $\left(\infty, \sigma_{0}\right)$-component $)$, and

$$
\pi: P_{\bar{x}} \longrightarrow G_{\bar{y}}
$$

the morphism induced by $\pi^{*}$.

Then the natural morphism

$$
W\left(\pi_{1}\left(X_{\bar{y}, \sigma_{0}}(\mathbb{C}), \bar{x}\right)\right) \stackrel{\eta_{\bar{x}}}{\longrightarrow} W_{\bar{x}}:=\operatorname{ker}(\pi)
$$

is an isomorphism.

c) If $Y^{\prime}=Y$, then $i$ defines a section of $\pi: P_{\bar{x}} \rightarrow G_{\bar{y}}$, and the functor

$$
\begin{aligned}
\pi-U M S_{\mathbb{Q}}^{s}(X) & \longrightarrow\left\{\begin{array}{l}
\text { objects } \mathbf{W} \text { of } M S_{\mathbb{Q}}^{s}(Y) \\
\text { together with a morphism of } \\
\text { algebras in } M S_{\mathbb{Q}}^{s}(Y) \\
i^{*} \mathcal{G} e n_{i} \rightarrow \underline{\operatorname{End}}_{\mathbb{Q}}(\mathbf{W})
\end{array}\right\}, \\
\mathbf{V} & \longmapsto i^{*} \mathbf{V}
\end{aligned}
$$

is an equivalence of categories.

d) If $Y^{\prime}=Y$, then the natural transformation of functors from $\pi-U M S_{\mathbb{Q}}^{s}(X)$ to $M S_{\mathbb{Q}}^{s}(Y)$

$$
\begin{aligned}
e v: \pi_{*} \underline{\operatorname{Hom}}\left(\mathcal{G e n}{ }_{i},-\right) & \longrightarrow i^{*}, \\
\varphi & \longmapsto\left(i^{*} \varphi\right)(1)
\end{aligned}
$$

is an isomorphism.

Proof: left to the reader.

q.e.d. 


\section{Remarks:}

a) Note that in the case of relative elliptic curves, the pro-sheaf $\mathcal{G} e n_{i}$ coincides with the "logarithmic sheaf" of [BL], 1.2. (Compare [BLp], $§ 1$ for the case of arbitrary curves, that are "unipotent $K(\pi, 1)$ s", i.e., unequal to $\mathbb{P}^{1}$.) This follows from 3.5.i) and [BL], 1.2.6.

Note that [BL], 1.2.10.v) coincides with 3.2.ii), 3.4.ii) and 3.6.c) here.

b) (compare $[\mathrm{BL}], 1.2 .10$.)

If we apply 3.5.i) to the pro-object $\mathcal{G} e n_{i} \hat{\otimes}_{\mathbb{Q}_{(l)}} \mathcal{G} e n_{i}$ and the section $1 \hat{\otimes} 1$, we get a comultiplication

$$
\mathcal{G} e n_{i} \longrightarrow \mathcal{G e n} \hat{\otimes}_{\mathbb{Q}_{(l)}} \mathcal{G} e n_{i},
$$

which is coassociative and cocommutative as follows from another application of 3.5.i).

If follows that $\mathcal{G} e n_{i}$ carries the structure of a cocommutative coalgebra, the counit being given by the augmentation morphism. Furthermore, $i^{*} \mathcal{G} e n_{i}$ carries the structure of an algebra, the unit being given by 1 .

Both structures are compatible, i.e., $i^{*} \mathcal{G} e n_{i}$ is equipped with a natural Hopf algebra structure. This is exactly the Hopf algebra structure corresponding to the tensor structure of

$$
\operatorname{Rep}_{F}\left(i^{*} \mathcal{G e n}_{i}\right):=\left\{\begin{array}{l}
\text { smooth sheaves } \mathbf{W} \text { on } Y \text { together with } \\
\text { a morphism of sheaves of algebras } \\
i^{*} \mathcal{G} e n_{i} \longrightarrow \underline{\operatorname{End}}_{F}(\mathbf{W})
\end{array}\right\}
$$

given by 3.2.ii), 3.4.ii) and 3.6.c) respectively.

c) Given a morphism $\pi: X \longrightarrow Y$ of type $(S)$, one may form the Poincaré groupoid sheaf, i.e., the $p r_{1}$-unipotent sheaf $\mathcal{G} e n_{\Delta}$ on $X \times_{Y} X$ associated to the "universal section" $\Delta$. It is certainly a more canonical object than the $\mathcal{G} e n_{i}$ associated to sections of $\pi$, and the main results of this section easily follow from the corresponding results for $\mathcal{G} e n_{\Delta}$. 


\section{$\S 4$ Families of unipotent $K(\pi, 1)$ s}

In this paragraph, we shall be concerned with the following problem: when is it possible to compute higher direct images of mixed smooth relatively unipotent sheaves within the category of such sheaves, i.e., by Hochschild cohomology? In doing so, we have to make use of certain categories of perverse sheaves (see [BBD], §2) and of Saito's theory of algebraic mixed Hodge modules ([S1], [S2]). Whenever we speak of perverse sheaves they will be formed with respect to the middle perversity. This means that we shall always have to deal with a shift of degree when comparing "usual" and perverse cohomology. We chose not to introduce any specific notation for the inclusion of the category of smooth sheaves into the category of all sheaves. However, this leads to the following slight complication: if for example $\mathbf{V}$ is an admissible variation of Hodge structure on $X$, then we consider $\mathbf{V}$ as an algebraic mixed Hodge module (see [S2], Theorem 3.27 and the remark following it). Its underlying perverse sheaf on $X(\mathbb{C})$ is the complex $\operatorname{For}(\mathbf{V})[\operatorname{dim} X]$, where $\operatorname{For}(\mathbf{V})$ denotes the local system underlying $\mathbf{V}$. We hope that these conventions won't lead to too much confusion.

\section{Definition:}

a) Let $\bar{X}$ be a pathwise connected topological space, $\bar{x} \in \bar{X}$ such that $\pi_{1}(\bar{X}, \bar{x})$ is finitely generated.

$\bar{X}$ is called a unipotent $K(\pi, 1)$ if the natural map

$$
H \cdot\left(W\left(\pi_{1}(\bar{X}, \bar{x})\right), \mathbb{Q}\right) \rightarrow H^{\cdot}(\bar{X}, \mathbb{Q})
$$

is an isomorphism.

(So if $\bar{X}$ is a $K(\pi, 1)$, then this is the case if and only if $\pi_{1}(\bar{X}, \bar{x})$ is pseudo-nilpotent.)

b) Let $\bar{X}$ be a pathwise connected scheme ([SGA4,III], Exp. IX, Définition 2.12) over an algebraically closed field $\bar{k}$ of characteristic $0, \bar{x}$ a geometric point such that $\pi_{1}(\bar{X}, \bar{x})$ is topologically finitely generated.

$\bar{X}$ is called a unipotent $l-K(\pi, 1)$ if the natural map

$$
H \cdot\left(W_{l}\left(\pi_{1}(\bar{X}, \bar{x})\right), \mathbb{Q}_{l}\right) \longrightarrow H_{\dot{e} t}\left(\bar{X}, \mathbb{Q}_{l}\right)
$$

is an isomorphism. 
Remark: We chose not to follow the terminology of $[\mathrm{CH}]$ and [H1], where a topological space as in a) is called a rational $K(\pi, 1)$.

Lemma 4.1: If $\bar{k}$ can be embedded into $\mathbb{C}$, and if $\bar{X} / \bar{k}$ is connected and of finite type, then for any embedding $\bar{k} \hookrightarrow \mathbb{C}$ such that $\pi_{1}(\bar{X}(\mathbb{C}), \bar{x})$ is finitely generated, $\bar{X}$ is a unipotent $l-K(\pi, 1)$ if and only if $\bar{X}(\mathbb{C})$ is a unipotent $K(\pi, 1)$.

Proof: By [SGA1], Exp. XII, Corollaire $5.2, \pi_{1}(\bar{X}, \bar{x})$ is the profinite completion of $\pi_{1}(\bar{X}(\mathbb{C}), \bar{x})$. So by 2.3 and the remark following 1.10 , the left hand sides of a) and b) coincide after tensoring a) with $\mathbb{Q}_{l}$.

Similarly, one uses [FK], Theorem 11.6 for the right hand sides.

q.e.d.

Since all the schemes occurring in this paragraph are of the type considered in the lemma, we shall also simply speak of unipotent $K(\pi, 1)$ s, the condition being checked at any prime number $l$.

From now on, it will happen frequently that definitions, theorems or proofs are "formally identical" in the Hodge theoretic and the $\lambda$-adic setting.

In order to make the writing style more economical, and also to make clear that the conclusions really are purely formal once we have a theory of sheaves satisfying a certain set of axioms, we fix the following rules:

whenever an area of paper is divided by a vertical bar:

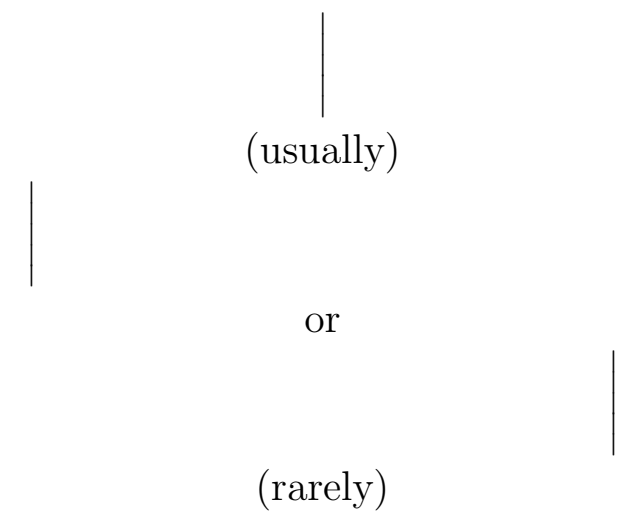

the text on the left of it will concern the Hodge-theoretic setting, while the text on the right will deal with the $\lambda$-adic setting. This understood, we let

$$
\begin{array}{l|l}
k:=\mathbb{C}, & k:=\text { a number field }, \\
F \subset \mathbb{R} \text { a subfield, } & \begin{array}{l}
l:=\text { a fixed prime number, } \\
F / \mathbb{Q}_{l} \text { finite or } F=\overline{\mathbb{Q}_{l}},
\end{array}
\end{array}
$$

$\pi: X \longrightarrow Y$ a morphism of type $(S)$ between schemes over $k$, 


$$
\begin{array}{l|l}
\bar{X}:=X(\mathbb{C}), & \bar{X}:=X \otimes_{k} \bar{k}, \\
\bar{Y}:=Y(\mathbb{C}) \text { as topological spaces, } & \bar{Y}:=Y \otimes_{k} \bar{k}, \\
\bar{\pi}: \bar{X} \rightarrow \bar{Y}, & \bar{\pi}: \bar{X} \rightarrow \bar{Y},
\end{array}
$$

$x \in X(\bar{k}), y:=\pi(x)$.

We continue to assume that after a finite étale covering $Y^{\prime} \rightarrow Y, \pi$ admits a section

$$
i: Y^{\prime} \rightarrow X^{\prime}:=X \times_{Y} Y^{\prime}
$$

sending a pre-image of $y$ to one of $x$.

The hypothesis that $Y$, hence also $X$, be geometrically connected is not really necessary. It just serves to make applicable the Tannakian formalism.

$$
\begin{array}{rl|l}
\operatorname{Sh}^{s}(Y):= & \operatorname{Var}_{F}(Y), \\
\operatorname{Sh}_{\pi}^{s}(X):= & \pi-U \operatorname{Var}_{F}(X), \\
\operatorname{Sh}^{s}(\bar{Y}):= & \text { the category of local } \\
& \text { systems of } F \text {-vector } \\
& \text { spaces on } \bar{Y}, \\
\operatorname{Sh}_{\pi}^{s}(X):= & \operatorname{Et}_{F}^{l, m}(Y), \\
\operatorname{Sh}^{s}(\bar{Y}):= & \text { the category of lisse } \\
& \\
& \text { constructible } \\
& F \text {-sheaves on } \bar{Y}, \\
\operatorname{Sh}_{\bar{\pi}}^{s}(\bar{X}):= & \text { the category of } \\
& \bar{\pi} \text {-unipotent local } \\
& \text { systems of } F \text {-vector } \\
& \text { spaces on } \bar{X} .
\end{array}
$$

Each of these categories is naturally contained in one of the following:

$$
\begin{aligned}
\operatorname{Sh}(Y) & :=\operatorname{MHM}_{F}(Y), \\
\operatorname{Sh}(X) & :=\operatorname{MHM}_{F}(X), \\
\operatorname{Sh}(\bar{Y}) & :=\operatorname{Perv}_{F}(\bar{Y}), \\
\operatorname{Sh}(\bar{X}) & :=\operatorname{Perv}_{F}(\bar{X}) .
\end{aligned}
$$

$$
\begin{aligned}
\operatorname{Sh}(Y) & :=\operatorname{Perv}_{F}^{m}(Y), \\
\operatorname{Sh}(X) & :=\operatorname{Perv}_{F}^{m}(X), \\
\operatorname{Sh}(\bar{Y}) & :=\operatorname{Perv}_{F}(\bar{Y}), \\
\operatorname{Sh}(\bar{X}) & :=\operatorname{Perv}_{F}(\bar{X}) .
\end{aligned}
$$

Here, $M H M_{F}$ denotes the category of algebraic mixed $F$-Hodge modules ([S2], $\S 4)$. Perv ${ }_{F}$ denotes the category of perverse sheaves on the topological space underlying a complex manifold ([BBD], 2.1) or on a smooth scheme over an algebraically closed field of characteristic zero ([BBD], 2.2). 
In order to define $\operatorname{Perv}_{F}^{m}(Y)$, we proceed as follows:

a constructible $F$-sheaf $\mathbf{V}$ on $Y$ is called mixed if it can be extended to a separated scheme of finite type $\mathfrak{Y} \rightarrow \operatorname{Spec}\left(o_{S}\right)$ such that the extension is mixed in the sense of [D3], Définition 1.2.2. $\operatorname{Et}_{F}^{m}(Y)$ is the category of mixed constructible sheaves on $Y$. We define $D_{m}^{b}(Y, F)$ to be the full subcategory of $D_{c}^{b}(Y, F)$ of those complexes whose usual cohomology objects lie in $\operatorname{Et}_{F}^{m}(Y)$. By [D3], VI, the categories $D_{m}^{b}(-, F)$ are stable under the usual six functors.

Since only these functors are used to define the perverse $t$-structure on $D_{c}^{b}(Y, F)$ (see in particular [BBD], Proposition 2.1.3 and Théorème 1.4.10), we may proceed as in [BBD], 5.1 and define $\operatorname{Perv}_{F}^{m}(Y)$ as the heart of $D_{m}^{b}(Y, F)$. We have perverse cohomology functors

$$
\mathcal{H}^{q}: D_{m}^{b}(Y, F) \rightarrow \operatorname{Perv}_{F}^{m}(Y) .
$$

In particular we have the perverse higher direct images

$$
\begin{gathered}
\mathcal{H}^{q} \pi_{*}: \operatorname{Perv}_{F}^{m}(X) \hookrightarrow D_{m}^{b}(X, F) \stackrel{\pi_{*} \text { restricted to }}{\underset{D_{m}^{b}(X, F)}{\longrightarrow}} \\
\longrightarrow D_{m}^{b}(Y, F) \stackrel{\mathcal{H}^{q}}{\longrightarrow} \operatorname{Perv}_{F}^{m}(Y) .^{\dagger}
\end{gathered}
$$

Because $\pi$ is of type $(S)$, up to a shift of degree, $\mathcal{H}^{q} \pi_{*}$ restricted to $\operatorname{Et}_{F}^{l, m}(X) \subset \operatorname{Perv}_{F}^{m}(X)$, can be computed via the ordinary higher direct image, which is in fact what we are always going to do.

Note that this definition should only be seen as a very modest approximation of what one might consider to be "the right one". As suggested by Saito's definition ([S2], 2.1) one should start by using a filtered category of complexes of constructible sheaves such that the filtration induces up to a shift the weight filtration on the mixed cohomology objects. However, the aim of this paragraph is only to show that under a condition on the fibres of $\bar{\pi}$, the higher direct image $\mathcal{H}^{q} \pi_{*}$, when restricted to $\operatorname{Sh}_{\pi}^{s}(X)$, can be calculated within $\operatorname{Sh}_{\pi}^{s}(X)$. For this, we just need a "surrounding triangulated category" of $\mathrm{Sh}_{\pi}^{s}(X)$ in $D_{c}^{b}(X, F)$. We could even have chosen $D_{c}^{b}(X, F)$. But we definitely feel that for less special $\pi$ as considered here, one should use perverse (as opposed to usual) higher direct images. In any case, as soon as the correct definition of Perv ${ }_{F}^{m}$ is found, Theorem 4.3 below will hold for $\operatorname{Sh}_{\pi}^{s}(X) \cap \operatorname{Perv}_{F}^{m}(X)$ if we manage to show that $\mathcal{G} e n_{i}$ lies in

\footnotetext{
${ }^{\dagger} \mathrm{As}$ in $[\mathrm{BBD}]$, we denote by $\pi_{*}, \pi^{*}, \mathcal{H}$ om etc. the respective functors on the derived category of mixed sheaves.
} 
pro-Perv ${ }_{F}^{m}(X)$ and if the natural functor

$$
\operatorname{Perv}_{F}^{m}(X) \longrightarrow D_{c}^{b}(X, F)
$$

is compatible with $\pi_{*}$.

We call sheaves on $\bar{Y}$ and $\bar{X}$ topological sheaves while referring to sheaves on $Y$ and $X$ simply as "sheaves". So we have natural forgetful functors associating to a sheaf its underlying topological sheaf. In the case of $\operatorname{Sh}(Y)$ and $\operatorname{Sh}(X)$, they are compatible with $\mathcal{H}^{q} \pi_{*}$ and $\mathcal{H}^{q} \bar{\pi}_{*}$ :

$$
\begin{array}{ll}
\operatorname{Sh}(X) \longrightarrow & \operatorname{Sh}(\bar{X}) \\
\mathcal{H}^{q} \pi_{*} \downarrow & \downarrow \mathcal{H}^{q} \bar{\pi}_{*} \\
\operatorname{Sh}(Y) \longrightarrow & \operatorname{Sh}(\bar{Y})
\end{array}
$$

commutes, as follows from

$$
\begin{array}{l|l}
\text { [S2], Theorem 4.3. } & \begin{array}{l}
\text { smooth base change ([SGA4,III], Exp. XVI, Corol- } \\
\text { laire 1.2). }
\end{array}
\end{array}
$$

An analogous statement holds for $\operatorname{Sh}^{s}(Y)$ and $\operatorname{Sh}_{\pi}^{s}(X)$ :

\section{Lemma 4.2:}

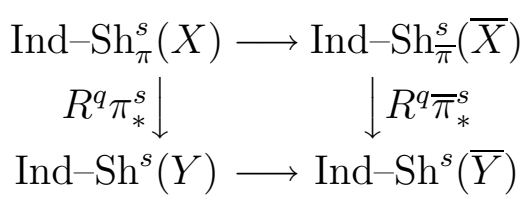

commutes.

Here, $R^{q} \pi_{*}^{s}$ is the $q$-th higher direct image, computed in $\operatorname{Ind}_{-} \operatorname{Sh}_{\pi}^{s}(X)$, which, being the ind-category of a neutral Tannakian category, has enough injectives (compare [Ho], §2). Similarly for $R^{q} \bar{\pi}_{*}^{s}$.

Proof: $R^{q} \pi_{*}^{s}$ is calculated using cohomology of $W_{\bar{x}}, R^{q} \bar{\pi}_{*}^{s}$ is calculated using cohomology of $W_{(l)}\left(\bar{X}_{\bar{y}}, \bar{x}\right)$. The groups coincide by

Corollary 3.4.i).

| Corollary 3.2.i).

q.e.d.

In particular, if $H \cdot\left(W_{\bar{x}}, \mathbb{Q}_{(l)}\right)$ is finite-dimensional, we have the same commutative diagram without the prefixes "Ind".

Slightly generalizing the notion recalled at the beginning of the paragraph, we define: 
Definition: Let $\bar{X}$ be a pathwise connected topological $\mid$ pathwise connected scheme over an space, $\bar{x} \in \bar{X}$ such that $\pi_{1}(\bar{X}, \bar{x})$ is finitely generated. algebraically closed field $\bar{k}$ of characteristic $0, \bar{x}$ a geometric point such that $\pi_{1}(\bar{X}, \bar{x})$ is topologically finitely generated.

$\bar{X}$ is called a unipotent $(l-) K\left(\pi, 1, \leq q_{0}\right)$ if the natural map

$$
H^{q}\left(W\left(\pi_{1}(\bar{X}, \bar{x})\right), M\right) \rightarrow H^{q}(\bar{X}, M) \mid H^{q}\left(W_{l}\left(\pi_{1}(\bar{X}, \bar{x})\right), M\right) \rightarrow H_{e t}^{q}(\bar{X}, M)
$$

is an isomorphism for any $q \leq q_{0}$, and any unipotent

$$
\text { local system of } \mathbb{Q} \text {-vector spaces } \quad \mid \text { lisse constructible } \mathbb{Q}_{l} \text {-sheaf }
$$

$M$ on $\bar{X}$.

So a sufficient condition is that the above map for $M=\mathbb{Q}_{(l)}$ be bijective for $q \leq q_{0}$ and injective for $q=q_{0}+1$.

By [Hub], Theorem 2.6, the system $\left(\left.\left(R^{q} \pi_{*}^{s}\right)\right|_{\mathrm{Sh}_{\pi}^{s}(X)}\right)_{q \in \mathbb{N}_{0}}$ is the cohomological derived functor of $\left.\pi_{*}^{s}\right|_{\mathrm{Sh}_{\pi}^{s}(X)}$. This guarantees the existence of the transformation of functors in the following

Theorem 4.3: Assume in addition to the hypothesis on $\pi$ already made, that the fibres of $\bar{\pi}$ are unipotent $K\left(\pi, 1, \leq q_{0}\right)$ s. Let $q \leq q_{0}, d:=\operatorname{dim} X-\operatorname{dim} Y$. Then the natural transformation of functors on $\mathrm{Sh}_{\pi}^{s}(X)$

$$
\left.R^{q} \pi_{*}^{s} \longrightarrow\left(\mathcal{H}^{q-d} \pi_{*}\right)\right|_{\mathrm{Sh}_{\pi}^{s}(X)}
$$

is an isomorphism.

Proof: Since the forgetful functors are exact and faithful, it suffices to show that the assertion holds on the level of topological sheaves. Remember that because $\pi$ is of type $(S)$, perverse higher direct images coincide, up to shift, with usual higher direct images, when evaluated on smooth topological sheaves. $R^{q} \bar{\pi}_{*}$ transforms smooth topological sheaves into smooth topological sheaves and satisfies base change. So the result follows from the definition of unipotent $K\left(\pi, 1, \leq q_{0}\right) \mathrm{s}$.

q.e.d.

It should be possible to define a category $M S_{\mathbb{Q}}(X)$ of mixed systems of constructible sheaves on a separated scheme $X$ of finite type over a number field 
$k$, and prove an analogue of 4.3 in this setting. Of course we are confident that once the "real" category of mixed motivic sheaves is found, the above proof will carry over without difficulties.

Theorem 4.3 allows us to compute $\pi_{*}\left(\mathcal{G} e n_{i}\right)$ under rather restrictive hypotheses on the fibres of $\bar{\pi}$. Note however that these assumptions are fulfilled if we consider the projection of a mixed Shimura variety (see $[\mathrm{P}]$ ) to the underlying pure Shimura variety.

Corollary 4.4: Let $d:=\operatorname{dim} X-\operatorname{dim} Y$ be the relative dimension of $\pi$. Assume that $W_{\bar{x}}$ is algebraic of dimension $N$ and that the fibres of $\bar{\pi}$ are unipotent $K(\pi, 1) \mathrm{s}$.

Consider the augmentation morphism

$$
\varepsilon: \mathcal{G} e n_{i} \longrightarrow \mathbb{Q}_{(l)}(0) .
$$

$\pi_{*}(\varepsilon)$ factors over an isomorphism

$$
\pi_{*}\left(\mathcal{G e n}_{i}\right) \stackrel{\sim}{\longrightarrow} \Lambda^{N}\left(\operatorname{Lie}\left(W_{\bar{x}}\right)\right)^{\vee}[-N+d]
$$

which is the unique map making the following diagram commutative:

$$
\begin{aligned}
\mathcal{H}^{N-d} \pi_{*}\left(\mathbb{Q}_{(l)}(0)\right)[-N+d] & \longrightarrow \pi_{*}\left(\mathbb{Q}_{(l)}(0)\right) \\
\imath \downarrow & \uparrow \pi_{*}(\varepsilon) \\
\Lambda^{N}\left(\operatorname{Lie}\left(W_{\bar{x}}\right)\right)^{\vee}[-N+d] & \stackrel{\sim}{\longleftarrow} \pi_{*}\left(\mathcal{G e n} n_{i}\right)
\end{aligned}
$$

More precisely, write

$$
\mathcal{G} e n_{i}=\lim _{n \in \mathbb{N}} \mathcal{G} e n_{i}^{(n)}
$$

where the $\mathcal{G} e n_{i}^{(n)}$ are smooth quotients of finite rank of $\mathcal{G} e n_{i}$, and let $\varepsilon^{(n)}, n \gg 0$ be the augmentations.

For any $q$, consider the projective system

$$
\left(\mathcal{H}^{q} \pi_{*}\left(\mathcal{G} e n_{i}^{(n)}\right)\right)_{n \in \mathbb{N}} .
$$

Then, for $q \neq N-d$, this system is ML-zero, i.e., for any $n$ there is a positive integer $m(q, n) \in \mathbb{N}$ such that the transition morphism

$$
\mathcal{H}^{q} \pi_{*}\left(\mathcal{G} e n_{i}^{(n+m(q, n))}\right) \longrightarrow \mathcal{H}^{q} \pi_{*}\left(\mathcal{G} e n_{i}^{(n)}\right)
$$


is zero.

For $n \gg 0$, the morphism

$$
\mathcal{H}^{N-d} \pi_{*}\left(\varepsilon^{(n)}\right): \mathcal{H}^{N-d} \pi_{*}\left(\mathcal{G} e n_{i}^{(n)}\right) \longrightarrow \mathcal{H}^{N-d} \pi_{*}\left(\mathbb{Q}_{(l)}(0)\right)
$$

is surjective. The projective system

$$
\left(\operatorname{ker}\left(\mathcal{H}^{N-d} \pi_{*}\left(\varepsilon^{(n)}\right)\right)\right)_{n \gg 0}
$$

is ML-zero. Observe that the map

$$
\mathcal{H}^{N-d} \pi_{*}\left(\mathbb{Q}_{(l)}(0)\right)[-N+d] \longrightarrow \pi_{*}\left(\mathbb{Q}_{(l)}(0)\right)
$$

exists because $\mathcal{H}^{q} \pi_{*}\left(\mathbb{Q}_{(l)}(0)\right)=0$ for $q>N-d$ by 4.3. The Tannaka dual $G_{\bar{y}}$ of $\operatorname{Sh}^{s}(Y)$ acts on $\operatorname{Lie}\left(W_{\bar{x}}\right)$ via the section $i$ and conjugation.

The left vertical arrow is induced by the canonical isomorphism of 1.13.

Remarks: a) Readers irritated by the "wrong" shifts should recall that the $\mathcal{H}^{q} \pi_{*}$ correspond to perverse higher direct images of $\bar{\pi}$.

b) While $N$ equals the cohomological dimension of $W_{\bar{x}}$, which can be defined whenever the fibres of $\bar{\pi}$ are unipotent $K(\pi, 1)$ s, the statement " $\mathcal{H}^{N-d} \pi_{*}\left(\mathcal{G e n}_{i}\right)$ is of rank one" is in general false without the algebraicity assumption on $W_{\bar{x}}$.

For $Y=\operatorname{Spec}(k), X$ an incomplete curve over $k$ not containing $\mathbb{G}_{m, k}$, we have $N=d=1$, but the vector space underlying

$$
\mathcal{H}^{0} \pi_{*}\left(\mathbb{Q}_{(l)}(0)\right)=H^{1}\left(\bar{X}, \mathbb{Q}_{(l)}(0)\right)
$$

is of finite dimension greater or equal to two.

Furthermore, $\mathcal{H}^{0} \pi_{*}\left(\mathcal{G}_{e} n_{i}\right)$ surjects onto $\mathcal{H}^{0} \pi_{*}\left(\mathbb{Q}_{(l)}(0)\right)$.

Proof of Corollary 4.4: By 4.3, we have to consider $\left(H^{q}\left(W_{\bar{x}}, \mathcal{G} e n_{i}^{(n)}\right)\right)_{n \in \mathbb{N}}$. But this is what we already did in 1.12.b) and 1.13. Note that in our situation, the isomorphism of 1.13 , being canonical, is an isomorphism of $G_{\bar{y}}$-modules. q.e.d. 


\section{Index of Notations}

\begin{tabular}{|c|c|c|c|}
\hline $\operatorname{Rep}_{F}(P)$ & 1 & $I_{\infty, \sigma}$ & 24 \\
\hline $\operatorname{Vec}_{F}$ & 1 & $c_{\infty, \sigma}$ & 25 \\
\hline$\hat{\mathfrak{U}}(\operatorname{Lie} \bar{W})$ & 3 & $c_{D R, \sigma}$ & 25 \\
\hline $\operatorname{Rat}(W)$ & 5 & $U M S_{\mathbb{Q}}^{s}(X)$ & 26 \\
\hline$R(W)$ & 5 & $\mathcal{G} e n_{x, \infty, \sigma}$ & 27 \\
\hline$H^{\cdot}(W, X)$ & 5 & $\mathcal{G} e n_{x, D R}$ & 28 \\
\hline$T(W)$ & 8 & $\mathcal{G}_{e n_{x, l}}$ & 29 \\
\hline$H .(W, M)$ & 9 & $H^{1}(\bar{X}, \mathbb{Q}(0))$ & 30 \\
\hline $\mathbb{Q}[\pi]$ & 12 & $\pi-U \mathrm{Et}_{F}^{l, m}(X)$ & 31 \\
\hline $\mathbb{Q}[\pi]^{\wedge}$ & 12 & $\hat{\mathfrak{U}}_{\pi, \bar{x}}$ & 32 \\
\hline$W(\pi)$ & 12 & $\mathcal{G}_{e n}$ & 32 \\
\hline $\mathfrak{w}(\pi)$ & 12 & $\bar{\pi}$ & 34 \\
\hline$W_{l}(\hat{\pi})$ & 13 & $\operatorname{Var}_{F}(X)$ & 34 \\
\hline $\mathfrak{w}_{l}(\hat{\pi})$ & 13 & $\pi-U \operatorname{Var}_{F}(X)$ & 34 \\
\hline $\bar{X}$ & 15 & $\pi-U M S_{\mathbb{Q}}^{s}(X)$ & 38 \\
\hline $\mathbb{Q}-M H S$ & 15 & $\mathcal{G} e n_{i, \infty, \sigma}$ & 38 \\
\hline$M H_{\mathbb{Q}}$ & 15 & $\mathcal{G}_{e n_{i, D R}}$ & 38 \\
\hline$\hat{\mathfrak{U}}_{\bar{x}}$ & 15 & $\mathcal{G e n}_{i, l}$ & 38 \\
\hline$U \operatorname{Var}_{\mathbb{Q}}(X)$ & 16 & $\operatorname{Sh}^{s}(Y)$ & 43 \\
\hline $\mathcal{G} e n_{x}$ & 17 & $\mathrm{Sh}_{\pi}^{s}(X)$ & 43 \\
\hline $\mathrm{Et}_{F}^{l, m}(X)$ & 18 & $\operatorname{Sh}^{s}(\bar{Y})$ & 43 \\
\hline$U \mathrm{Et}_{F}^{l, m}(X)$ & 18 & $\operatorname{Sh}_{\bar{\pi}}^{s}(\bar{X})$ & 43 \\
\hline $\mathbb{Q}_{(l)}$ & 22 & $\operatorname{Sh}(Y)$ & 43 \\
\hline$M S_{\mathbb{Q}}^{s}(X)$ & 23 & $\operatorname{Sh}(\bar{Y})$ & 43 \\
\hline$I_{l, \bar{\sigma}}$ & 24 & $\mathcal{H}^{q} \pi_{*}$ & 44 \\
\hline$I_{D R, \sigma}$ & 24 & $\mathcal{H}^{q} \bar{\pi}_{*}$ & 45 \\
\hline$c_{\sigma}$ & 24 & $R^{q} \pi_{*}^{s}$ & EJ \\
\hline$c_{\sigma}^{*}$ & 24 & $R^{q} \bar{\pi}_{*}^{s}$ & 45 \\
\hline
\end{tabular}




\section{References}

[B] A.A. Beilinson, "Polylogarithm and Cyclotomic Elements", typewritten preprint, MIT 1989 or 1990.

[BBD] A.A. Beilinson, J. Bernstein, P. Deligne, "Faisceaux pervers", in B. Teissier, J.L. Verdier, "Analyse et Topologie sur les Espaces singuliers" (I), Astérisque 100, Soc. Math. France 1982.

[BD] A.A. Beilinson, P. Deligne, "Motivic Polylogarithm and Zagier Conjecture", preprint, 1992.

[BL] A.A. Beilinson, A. Levin, "The Elliptic Polylogarithm", in U. Jannsen, S.L. Kleiman, J.-P. Serre, "Motives", Proc. of Symp. in Pure Math. 55, Part II, AMS 1994, pp. 123-190.

[BLp] A.A. Beilinson, A. Levin, "Elliptic Polylogarithm", typewritten preliminary version of [BL], preprint, MIT 1992.

[BLpp] A.A. Beilinson, A. Levin, "Elliptic Polylogarithm", handwritten preliminary version of [BLp], June 1991.

[C] K.-T. Chen, "Iterated path integrals", Bull. AMS 83 (1977), pp. 831-879.

[CH] J.A. Carlson, R.M. Hain, "Extensions of Variations of Mixed Hodge Structure", in D. Barlet, H. Esnault, F. El Zein, J.L. Verdier, E. Viehweg, "Actes du Colloque de Théorie de Hodge, Luminy 1987", Astérisque 179-180, Soc. Math. France 1989, pp. 39-65.

[D1] P. Deligne, "Equations Différentielles à Points Singuliers Réguliers", LNM 163, Springer-Verlag 1970.

[D2] P. Deligne, "Théorie de Hodge, II", Publ. Math. IHES 40 (1971), pp. 5-57.

[D3] P. Deligne, "La Conjecture de Weil II", Publ. Math. IHES 52 (1981), pp. 313-428. 
[D4] P. Deligne, "Le Groupe Fondamental de la Droite Projective Moins Trois Points", in Y. Ihara, K. Ribet, J.-P. Serre, "Galois Groups over Q", Math. Sci. Res. Inst. Publ. 16, Springer-Verlag 1989, pp. $79-297$.

[DG] M. Demazure, P. Gabriel, "Groupes Algébriques", Tôme 1, North-Holland Publ. Comp. 1970.

[DM] P. Deligne, J.S. Milne, "Tannakian Categories", in P. Deligne, J.S. Milne, A. Ogus, K.-y. Shih, "Hodge Cycles, Motives, and Shimura varieties", LNM 900, Springer-Verlag 1982, pp. 101-228.

[EGAIV,3] A. Grothendieck, J. Dieudonné, "Etude locale des Schémas et des Morphismes de Schémas", Troisième Partie, Publ. Math. IHES 28 (1966).

[FK] E. Freitag, R. Kiehl, "Etale Cohomology and the Weil Conjecture", Erg. der Math. und ihrer Grenzgeb., Band 13, SpringerVerlag 1988.

[H1] R.M. Hain, "Algebraic Cycles and Extensions of Variations of Mixed Hodge Structure", in J.A. Carlson, C.H. Clemens, D.R. Morrison, "Complex Geometry and Lie Theory", Proc. of Symp. in Pure Math. 53, AMS 1991, pp. 175-221.

[H2] R.M. Hain, "The Geometry of the Mixed Hodge Structure on the Fundamental Group", in S.J. Bloch, "Algebraic Geometry Bowdoin 1985", Proc. of Symp. in Pure Math. 46, AMS 1987, pp. 247-282.

[Hi] H. Hironaka, "Resolutions of singularities of an algebraic variety over a field of characteristic zero", Ann. of Math. 79 (1964), pp. 109-326.

[Ho] G. Hochschild, "Cohomology of Algebraic Linear Groups", Illinois Jour. of Math. 5 (1961), pp. 492-519.

[Hub] A. Huber, "Calculation of Derived Functors via Ind-Categories", Jour. of Pure and Appl. Algebra 90 (1993), pp. 39-48. 
[Hum1] J.E. Humphreys, "Linear Algebraic Groups", GTM 21, SpringerVerlag 1987.

[Hum2] J.E. Humphreys, "Introduction to Lie Algebras and Representation Theory", GTM 9, Springer-Verlag 1980.

[HZ1] R.M. Hain, S. Zucker, "Unipotent variations of mixed Hodge structure", Inv. math. 88 (1987), pp. 83-124.

[HZ2] R.M. Hain, S. Zucker, "A Guide to Unipotent variations of mixed Hodge structure", in E. Cattani, F. Guillén, A. Kaplan, F. Puerta, "Hodge Theory. Proceedings, Sant Cugat, Spain, 1985", LNM 1246, Springer-Verlag 1987, pp. 92-106.

[J] U. Jannsen, "Mixed Motives and Algebraic $K$-Theory", LNM 1400, Springer-Verlag 1990.

[K] A.W. Knapp, "Lie groups, Lie algebras, and Cohomology", Mathematical Notes 34, Princeton Univ. Press 1988.

[Ka] M. Kashiwara, "A Study of Variation of Mixed Hodge Structure", Publ. RIMS, Kyoto Univ. 22 (1986), pp. 991-1024.

[P] R. Pink, "Arithmetical compactification of Mixed Shimura Varieties", thesis, Bonner Mathematische Schriften 1989.

[S1] Morihiko Saito, "Modules de Hodge Polarisables", Publ. RIMS, Kyoto Univ. 24 (1988), pp. 849-995.

[S2] Morihiko Saito, "Mixed Hodge Modules", Publ. RIMS, Kyoto Univ. 26 (1990), pp. 221-333.

[Sch] W. Schmid, "Variation of Hodge Structure: The Singularities of the Period Mapping", Inv. math. 22 (1973), pp. 211-319.

[SGA1] A. Grothendieck et al., "Revêtements Etales et Groupe Fondamental", LNM 224, Springer-Verlag 1971.

[SGA4,II] M. Artin, A. Grothendieck, J.L. Verdier et al., "Théorie des Topos et Cohomologie Etale des Schémas", Tôme 2, LNM 270, SpringerVerlag 1972. 
[SGA4,III] M. Artin, A. Grothendieck, J.L. Verdier et al., "Théorie des Topos et Cohomologie Etale des Schémas", Tôme 3, LNM 305, SpringerVerlag 1973.

[SGA4 1/2] P. Deligne et al., "Cohomologie Etale", LNM 569, Springer-Verlag 1977.

[Sp] E.H. Spanier, "Algebraic Topology", Springer-Verlag 1966.

[SZ] J. Steenbrink, S. Zucker, "Variation of mixed Hodge structure, I", Inv. math. 80 (1985), pp. 489-542.

[W] J. Wildeshaus, "Polylogarithmic Extensions on Mixed Shimura varieties", Schriftenreihe des Mathematischen Instituts der Universität Münster, 3. Serie, Heft 12, 1994.

[Wo1] Z. Wojtkowiak, "Cosimplicial objects in algebraic geometry", in "Algebraic K-theory and Algebraic Topology", Proceedings of the Lake Louise conference 1991 or 1992, Kluver Academic Publishers 1993, pp. 287-327.

[Wo2] Z. Wojtkowiak, "Cosimplicial objects in algebraic geometry II", preprint. 